\title{
RELATIVISTIC ROTATION OF THE RIGID BODY IN THE RODRIGUES - HAMILTON PARAMETERS: LAGRANGE FUNCTION AND EQUATIONS OF MOTION
}

\author{
V.V. Pashkevich and G.I. Eroshkin \\ Central (Pulkovo) Astronomical Observatory of RAS \\ Pulkovskoe shosse, 65/1, 196140, St. Petersburg, Russia \\ e-mail: pashvladvit@yandex.ru
}

\begin{abstract}
The main purposes of this research are to obtain Lagrange function for the relativistic rotation of the rigid body, which is generated by metric properties of Riemann space of general relativity and to derive the differential equations, determining the rigid body rotation in the terms of the Rodrigues - Hamilton parameters. The Lagrange function for the relativistic rotation of the rigid body is derived from the Lagrange function of the nonrotation point of masses system in the relativistic approximation.
\end{abstract}

Keywords: the rigid body rotation, Rodrigues - Hamilton parameters, Lagrange function, the relativistic approximation.

\section{BASIC EQUATIONS}

The construction of the Lagrange function for the case when a certain set of point masses $m_{n_{\alpha}}=d m_{n}$ from the whole system of point masses $m_{i}$ forms an 'absolutely rigid body' $m_{n}$ (presented in Figures $1-2$ ) in such a way that the condition $\Delta_{n_{p} n_{q}} \equiv$ const (Suslov, 1946) (Figure 1) holds for any point masses $m_{n_{p}}$ and $m_{n_{q}}$ from the set $m_{n_{\alpha}}$. In this case, the body $m_{n}$ can rotate around its own center of mass with angular velocity $|\bar{\omega}| \geq 0$, the remaining point bodies $m_{j}$ from the set $m_{i}$ do not rotate. Here $m_{j}$ is the mass of the $j$-th point; $O_{n}$ is the center of masses rigid body $m_{n} ; B$ is barycentre of a masses point system; $B \bar{I}_{1} \bar{I}_{2} \bar{I}_{3}$ is barycentric coordinate system; $O_{n} \bar{I}_{1} \bar{I}_{2} \bar{I}_{3}$ is a coordinate system of the rigid body, whose axes are parallel to the axes of the barycentric coordinate system; $O_{n} \overline{i_{1}} \dot{i}_{2} \dot{\vec{L}}_{3}$ is a coordinate system of the rigid body $m_{n}$, whose axes are principal axes of inertia of this body; $d m_{n}$ (Figure 2 ) is the element of a set of point of masses of the rigid body $m_{n} ; \bar{\rho}$ is radius vector of the mass element $d m_{n} ; \bar{R}_{n}^{*}, \bar{\Delta}_{n j}^{*}$ are barycentric and $m_{j}$ body vectors of the mass elements $d m_{n}$ respectively; $\bar{R}_{n}, \bar{\Delta}_{n j}$ are barycentric and $m_{j}$ body vectors of the center of masses rigid body $m_{n}$, respectively; $\bar{R}_{j}$ is barycentric vector of the point bodies $m_{j}$. The coordinate system $O_{n} \overline{i_{1} i_{2}} \bar{i}_{3}$ of the rigid body $m_{n}$ rotates relatively to barycentric coordinate system with angular velocity $\bar{\omega}$. 


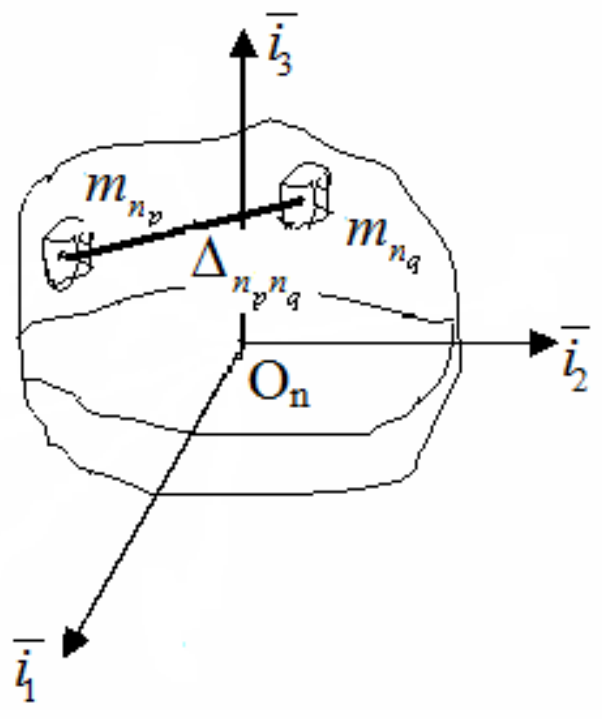

Fig. 1.

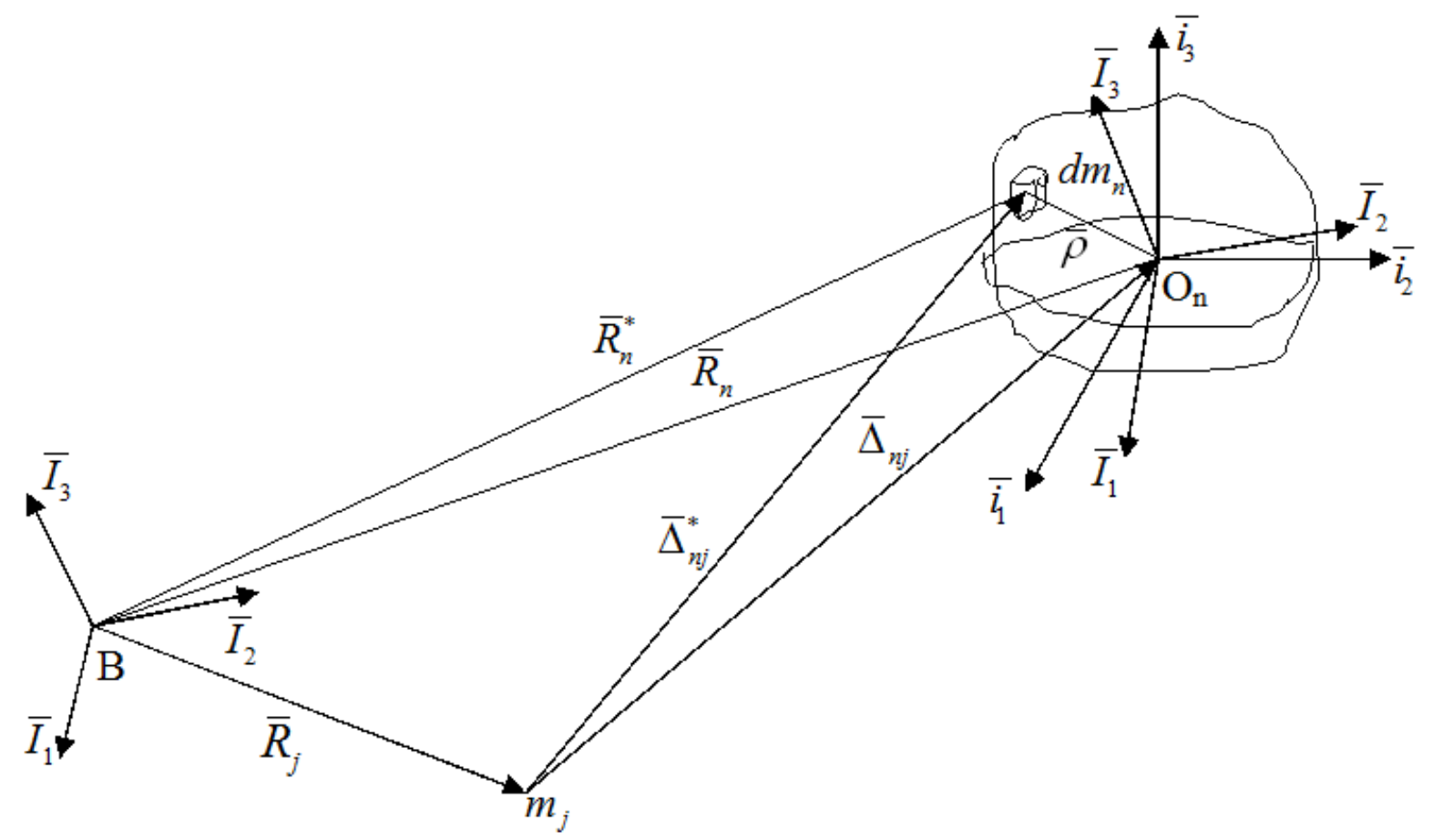

Fig. 2.

The radius vectors of the center of masses $O_{n}$ of the rigid body $m_{n}$ in coordinate system $B \bar{I}_{1} \bar{I}_{2} \bar{I}_{3}$ and in the projections on the axes system $O_{n} \bar{i}_{1} \dot{i}_{2} \overline{\dot{L}}_{3}$ have forms: $\bar{R}_{n}=X_{n} \bar{I}_{1}+Y_{n} \bar{I}_{2}+Z_{n} \bar{I}_{3}$ and $\bar{R}_{n}=x_{n} \bar{i}_{1}+y_{n} \bar{i}_{2}+z_{n} \overline{\dot{j}}_{3}$, respectively. The radius vectors of the mass element $d m_{n}$ of the rigid body $m_{n}$ in barycentric coordinate system $B \bar{I}_{1} \bar{I}_{2} \bar{I}_{3}$ and in the projections on the axes system $O_{n}{\overline{i_{1}}}_{2} \bar{i}_{3}$ have forms: $\bar{R}_{n}^{*}=X_{n}^{*} \bar{I}_{1}+Y_{n}^{*} \bar{I}_{2}+Z_{n}^{*} \bar{I}_{3}$ and $\bar{R}_{n}^{*}=x_{n}^{*} \overline{\dot{i}}_{1}+y_{n}^{*}{\overline{i_{2}}}_{2}+z_{n}^{*} \bar{i}_{3}$, respectively. The radius vector of the mass element $d m_{n}$ of the rigid body $m_{n}$ in the coordinate system $O_{n} \overline{\dot{i}_{1} \dot{i}_{2}} \dot{\vec{\zeta}}_{3}$ has a form: $\bar{\rho}=\xi \overline{\dot{i}_{1}}+\eta \overline{\dot{i}_{2}}+\zeta \overline{\dot{i}_{3}}$, where $\xi, \eta, \zeta$ are constants. Relation of these three radius vectors has a form: $\bar{R}_{n}^{*}=\bar{R}_{n}+\bar{\rho}$. 
After termwise differentiation with respect to time, the ratio of the velocity vectors was obtained: $\dot{\bar{R}}_{n}^{*}=\dot{\bar{R}}_{n}+\dot{\bar{\rho}}$. Velocity of vector of the mass element $d m_{n}$ of the rigid body $m_{n}$ in coordinate system $O_{n} \bar{I}_{1} \bar{I}_{2} \bar{I}_{3}$ has a form (Euler formula): $\dot{\bar{\rho}}=\bar{\omega} \times \bar{\rho} \Rightarrow \dot{\bar{R}}_{n}^{*}=\dot{\bar{R}}_{n}+\bar{\omega} \times \bar{\rho}$. Here and further sign $\times$ stands for the vector product; $\bar{\omega}=\omega_{1} \bar{i}_{1}+\omega_{2} \bar{i}_{2}+\omega_{3} \bar{i}_{3} ; \omega_{1}, \omega_{2}, \omega_{3}$ are the projections of angular velocity vector on the principal axes of the inertias of the rigid body $m_{n} ; \bar{H}_{n}=A_{n} \omega_{1} \bar{i}_{1}+B_{n} \omega_{2} \bar{i}_{2}+C_{n} \omega_{3} \bar{i}_{3}$ is the angular momentum vector of the rotational motion of the rigid body $m_{n} ; A_{n}, B_{n}, C_{n}$ are the principal moments of inertia of the second order of the rigid body $m_{n}$.

$$
A_{n}=\int_{m_{n}}\left(\eta^{2}+\zeta^{2}\right) d m_{n} ; B_{n}=\int_{m_{n}}\left(\zeta^{2}+\xi^{2}\right) d m_{n} ; C_{n}=\int_{m_{n}}\left(\xi^{2}+\eta^{2}\right) d m_{n} ; d m_{n}=p(\xi, \eta, \zeta) d \xi d \eta d \zeta ;
$$

$p(\xi, \eta, \zeta)$ is mass distribution function of the body $m_{n}$.

In the particular case when the body $m_{n}$ is a homogeneous triaxial ellipsoid with semiaxes $a, b, c$, its moments of inertia are determined by the following expressions (MacMillan,1936):

$$
A_{n}=\frac{m_{n}}{5}\left(b^{2}+c^{2}\right), \quad B_{n}=\frac{m_{n}}{5}\left(c^{2}+a^{2}\right), \quad C_{n}=\frac{m_{n}}{5}\left(a^{2}+b^{2}\right) .
$$

It is easy to see that when $a, b, c \rightarrow 0$, then $A_{n}, B_{n}, C_{n} \rightarrow 0$.

The velocity vector of the mass element $d m_{n}$ in the projections on the axes of the rotating coordinate system $O_{n} \overline{\dot{i}_{1}} \bar{i}_{2}$ has a form:

$$
\left(\begin{array}{lll}
a_{11} & a_{12} & a_{13} \\
a_{21} & a_{22} & a_{23} \\
a_{31} & a_{32} & a_{33}
\end{array}\right)\left(\begin{array}{l}
\dot{X}_{n}^{*} \\
\dot{Y}_{n}^{*} \\
\dot{Z}_{n}^{*}
\end{array}\right)=\left(\begin{array}{lll}
a_{11} & a_{12} & a_{13} \\
a_{21} & a_{22} & a_{23} \\
a_{31} & a_{32} & a_{33}
\end{array}\right)\left(\begin{array}{l}
\dot{X}_{n} \\
\dot{Y}_{n} \\
\dot{Z}_{n}
\end{array}\right)+\left(\begin{array}{ccc}
0 & -\omega_{3} & \omega_{2} \\
\omega_{3} & 0 & -\omega_{1} \\
-\omega_{2} & \omega_{1} & 0
\end{array}\right)\left(\begin{array}{l}
\xi \\
\eta \\
\zeta
\end{array}\right)
$$

or

$$
\left(\begin{array}{c}
\dot{x}_{n}^{*} \\
\dot{y}_{n}^{*} \\
\dot{z}_{n}^{*}
\end{array}\right)=\left(\begin{array}{l}
\dot{x}_{n} \\
\dot{y}_{n} \\
\dot{z}_{n}
\end{array}\right)+\left(\begin{array}{ccc}
0 & -\omega_{3} & \omega_{2} \\
\omega_{3} & 0 & -\omega_{1} \\
-\omega_{2} & \omega_{1} & 0
\end{array}\right)\left(\begin{array}{l}
\xi \\
\eta \\
\zeta
\end{array}\right)
$$

Here and further $\left(a_{i j}\right)$ is transformation matrix. Besides

or equivalent formula

$$
\left(\begin{array}{c}
\dot{X}_{n}^{*} \\
\dot{Y}_{n}^{*} \\
\dot{Z}_{n}^{*}
\end{array}\right)=\left(\begin{array}{c}
\dot{X}_{n} \\
\dot{Y}_{n} \\
\dot{Z}_{n}
\end{array}\right)+\left(\begin{array}{lll}
a_{11} & a_{21} & a_{31} \\
a_{12} & a_{22} & a_{32} \\
a_{13} & a_{23} & a_{33}
\end{array}\right)\left(\begin{array}{ccc}
0 & -\omega_{3} & \omega_{2} \\
\omega_{3} & 0 & -\omega_{1} \\
-\omega_{2} & \omega_{1} & 0
\end{array}\right)\left(\begin{array}{l}
\xi \\
\eta \\
\zeta
\end{array}\right)
$$

$$
\left(\begin{array}{c}
\dot{X}_{n}^{*} \\
\dot{Y}_{n}^{*} \\
\dot{Z}_{n}^{*}
\end{array}\right)=\left(\begin{array}{c}
\dot{X}_{n} \\
\dot{Y}_{n} \\
\dot{Z}_{n}
\end{array}\right)+\left(\begin{array}{lll}
a_{11} & a_{21} & a_{31} \\
a_{12} & a_{22} & a_{32} \\
a_{13} & a_{23} & a_{33}
\end{array}\right)\left(\begin{array}{ccc}
0 & -\zeta & \eta \\
\zeta & 0 & -\xi \\
-\eta & \xi & 0
\end{array}\right)\left(\begin{array}{c}
\omega_{1} \\
\omega_{2} \\
\omega_{3}
\end{array}\right)
$$

In celestial mechanics the rotation of a celestial body about its center of mass is described as the rotation of its principal axes of inertia with respect to the non-rotating bodycentric coordinate system. As the variables of this problem, usually used four RodriguesHamilton parameters:

$$
\lambda_{0}=\cos \frac{\theta}{2} \cos \frac{\psi+\varphi}{2}, \lambda_{1}=\sin \frac{\theta}{2} \cos \frac{\psi-\varphi}{2}, \lambda_{2}=\sin \frac{\theta}{2} \sin \frac{\psi-\varphi}{2}, \lambda_{3}=\cos \frac{\theta}{2} \sin \frac{\psi+\varphi}{2}
$$


which are the functions of the Euler angles $\psi, \theta$ and $\varphi$. These parameters are bounded variables. It is very important for the numerical solution of the problem.

The transformation matrix and the angular velocity vector of the rigid body $m_{n}$ can be expressed in terms of the Rodrigues-Hamilton parameters:

$$
\begin{aligned}
K K & =\left(\begin{array}{cccc}
-\lambda_{0} & -\lambda_{1} & -\lambda_{2} & -\lambda_{3} \\
-\lambda_{1} & \lambda_{0} & \lambda_{3} & -\lambda_{2} \\
-\lambda_{2} & -\lambda_{3} & \lambda_{0} & \lambda_{1} \\
-\lambda_{3} & \lambda_{2} & -\lambda_{1} & \lambda_{0}
\end{array}\right)\left(\begin{array}{cccc}
-\lambda_{0} & -\lambda_{1} & -\lambda_{2} & -\lambda_{3} \\
-\lambda_{1} & \lambda_{0} & \lambda_{3} & -\lambda_{2} \\
-\lambda_{2} & -\lambda_{3} & \lambda_{0} & \lambda_{1} \\
-\lambda_{3} & \lambda_{2} & -\lambda_{1} & \lambda_{0}
\end{array}\right)= \\
& =\left(\begin{array}{cccc}
1 & 0 & 0 & 0 \\
0 & \lambda_{0}^{2}+\lambda_{1}^{2}-\lambda_{2}^{2}-\lambda_{3}^{2} & 2\left(\lambda_{0} \lambda_{3}+\lambda_{1} \lambda_{2}\right) & 2\left(\lambda_{1} \lambda_{3}-\lambda_{0} \lambda_{2}\right) \\
0 & 2\left(\lambda_{1} \lambda_{2}-\lambda_{0} \lambda_{3}\right) & \lambda_{0}^{2}-\lambda_{1}^{2}+\lambda_{2}^{2}-\lambda_{3}^{2} & 2\left(\lambda_{0} \lambda_{1}+\lambda_{2} \lambda_{3}\right) \\
0 & 2\left(\lambda_{0} \lambda_{2}+\lambda_{1} \lambda_{3}\right) & 2\left(\lambda_{2} \lambda_{3}-\lambda_{0} \lambda_{1}\right) & \lambda_{0}^{2}-\lambda_{1}^{2}-\lambda_{2}^{2}+\lambda_{3}^{2}
\end{array}\right)=\left(\begin{array}{cccc}
1 & 0 & 0 & 0 \\
0 & a_{11} & a_{12} & a_{13} \\
0 & a_{21} & a_{22} & a_{23} \\
0 & a_{31} & a_{32} & a_{33}
\end{array}\right) .
\end{aligned}
$$

The transformation inverse matrix in terms of the Rodrigues-Hamilton parameters has a form:

$$
\begin{aligned}
K^{-1} K^{-1} & =\left(\begin{array}{cccc}
-\lambda_{0} & -\lambda_{1} & -\lambda_{2} & -\lambda_{3} \\
-\lambda_{1} & \lambda_{0} & -\lambda_{3} & \lambda_{2} \\
-\lambda_{2} & \lambda_{3} & \lambda_{0} & -\lambda_{1} \\
-\lambda_{3} & -\lambda_{2} & \lambda_{1} & \lambda_{0}
\end{array}\right)\left(\begin{array}{cccc}
-\lambda_{0} & -\lambda_{1} & -\lambda_{2} & -\lambda_{3} \\
-\lambda_{1} & \lambda_{0} & -\lambda_{3} & \lambda_{2} \\
-\lambda_{2} & \lambda_{3} & \lambda_{0} & -\lambda_{1} \\
-\lambda_{3} & -\lambda_{2} & \lambda_{1} & \lambda_{0}
\end{array}\right)= \\
& =\left(\begin{array}{cccc}
1 & 0 & 0 & 0 \\
0 & \lambda_{0}^{2}+\lambda_{1}^{2}-\lambda_{2}^{2}-\lambda_{3}^{2} & 2\left(\lambda_{1} \lambda_{2}-\lambda_{0} \lambda_{3}\right) & 2\left(\lambda_{0} \lambda_{2}+\lambda_{1} \lambda_{3}\right) \\
0 & 2\left(\lambda_{0} \lambda_{3}+\lambda_{1} \lambda_{2}\right) & \lambda_{0}^{2}-\lambda_{1}^{2}+\lambda_{2}^{2}-\lambda_{3}^{2} & 2\left(\lambda_{2} \lambda_{3}-\lambda_{0} \lambda_{1}\right) \\
0 & 2\left(\lambda_{1} \lambda_{3}-\lambda_{0} \lambda_{2}\right) & 2\left(\lambda_{0} \lambda_{1}+\lambda_{2} \lambda_{3}\right) & \lambda_{0}^{2}-\lambda_{1}^{2}-\lambda_{2}^{2}+\lambda_{3}^{2}
\end{array}\right)=\left(\begin{array}{cccc}
1 & 0 & 0 & 0 \\
0 & a_{11} & a_{21} & a_{31} \\
0 & a_{12} & a_{22} & a_{32} \\
0 & a_{13} & a_{23} & a_{33}
\end{array}\right) .
\end{aligned}
$$

The angular velocity vector of the rigid body $m_{n}$ in terms of the Rodrigues-Hamilton parameters has a form:

$$
\left(\begin{array}{c}
0 \\
\omega_{1} \\
\omega_{2} \\
\omega_{3}
\end{array}\right)=2\left(\begin{array}{cccc}
-\lambda_{0} & -\lambda_{1} & -\lambda_{2} & -\lambda_{3} \\
-\lambda_{1} & \lambda_{0} & \lambda_{3} & -\lambda_{2} \\
-\lambda_{2} & -\lambda_{3} & \lambda_{0} & \lambda_{1} \\
-\lambda_{3} & \lambda_{2} & -\lambda_{1} & \lambda_{0}
\end{array}\right)\left(\begin{array}{c}
\dot{\lambda}_{0} \\
\dot{\lambda}_{1} \\
\dot{\lambda}_{2} \\
\dot{\lambda}_{3}
\end{array}\right)\left(\begin{array}{c}
0 \\
\omega_{X} \\
\omega_{Y} \\
\omega_{Z}
\end{array}\right)=2\left(\begin{array}{cccc}
-\lambda_{0} & -\lambda_{1} & -\lambda_{2} & -\lambda_{3} \\
-\lambda_{1} & \lambda_{0} & -\lambda_{3} & \lambda_{2} \\
-\lambda_{2} & \lambda_{3} & \lambda_{0} & -\lambda_{1} \\
-\lambda_{3} & -\lambda_{2} & \lambda_{1} & \lambda_{0}
\end{array}\right)\left(\begin{array}{c}
\dot{\lambda}_{0} \\
\dot{\lambda}_{1} \\
\dot{\lambda}_{2} \\
\dot{\lambda}_{3}
\end{array}\right) .
$$

Here $\omega_{1}, \omega_{2}, \omega_{3}$ are the components of the vector $\bar{\omega}$ in the coordinate system $O_{n} \overline{i_{1}} \bar{i}_{2} \bar{i}_{3}$; $\omega_{X}, \omega_{Y}, \omega_{Z}$ are the projections of this vector on the axes of the non-rotating $O_{n} \bar{I}_{1} \bar{I}_{2} \bar{I}_{3}$ reference system.

Relation between these projections in the different coordinate systems is: 


$$
\begin{aligned}
& \left(\begin{array}{c}
0 \\
\omega_{1} \\
\omega_{2} \\
\omega_{3}
\end{array}\right)=\left(\begin{array}{cccc}
1 & 0 & 0 & 0 \\
0 & \lambda_{0}^{2}+\lambda_{1}^{2}-\lambda_{2}^{2}-\lambda_{3}^{2} & 2\left(\lambda_{0} \lambda_{3}+\lambda_{1} \lambda_{2}\right) & 2\left(\lambda_{1} \lambda_{3}-\lambda_{0} \lambda_{2}\right) \\
0 & 2\left(\lambda_{1} \lambda_{2}-\lambda_{0} \lambda_{3}\right) & \lambda_{0}^{2}-\lambda_{1}^{2}+\lambda_{2}^{2}-\lambda_{3}^{2} & 2\left(\lambda_{0} \lambda_{1}+\lambda_{2} \lambda_{3}\right) \\
0 & 2\left(\lambda_{0} \lambda_{2}+\lambda_{1} \lambda_{3}\right) & 2\left(\lambda_{2} \lambda_{3}-\lambda_{0} \lambda_{1}\right) & \lambda_{0}^{2}-\lambda_{1}^{2}-\lambda_{2}^{2}+\lambda_{3}^{2}
\end{array}\right)\left(\begin{array}{c}
0 \\
\omega_{X} \\
\omega_{Y} \\
\omega_{Z}
\end{array}\right)= \\
& =K K\left(\begin{array}{c}
0 \\
\omega_{X} \\
\omega_{Y} \\
\omega_{Z}
\end{array}\right)=\left(\begin{array}{cccc}
1 & 0 & 0 & 0 \\
0 & a_{11} & a_{12} & a_{13} \\
0 & a_{21} & a_{22} & a_{23} \\
0 & a_{31} & a_{32} & a_{33}
\end{array}\right)\left(\begin{array}{c}
0 \\
\omega_{X} \\
\omega_{Y} \\
\omega_{Z}
\end{array}\right)
\end{aligned}
$$

The velocity vector in terms of the Rodrigues-Hamilton parameters is:

$$
\begin{aligned}
& \left(\begin{array}{c}
0 \\
\dot{X}_{n}^{*} \\
\dot{Y}_{n}^{*} \\
\dot{Z}_{n}^{*}
\end{array}\right)=\left(\begin{array}{c}
0 \\
\dot{X}_{n} \\
\dot{Y}_{n} \\
\dot{Z}_{n}
\end{array}\right)+\left(\begin{array}{cccc}
1 & 0 & 0 & 0 \\
0 & a_{11} & a_{21} & a_{31} \\
0 & a_{12} & a_{22} & a_{32} \\
0 & a_{13} & a_{23} & a_{33}
\end{array}\right)\left(\begin{array}{cccc}
1 & 0 & 0 & 0 \\
0 & 0 & -\omega_{3} & \omega_{2} \\
0 & \omega_{3} & 0 & -\omega_{1} \\
0 & -\omega_{2} & \omega_{1} & 0
\end{array}\right)\left(\begin{array}{l}
0 \\
\xi \\
\eta \\
\zeta
\end{array}\right)= \\
& =\left(\begin{array}{c}
0 \\
\dot{X}_{n} \\
\dot{Y}_{n} \\
\dot{Z}_{n}
\end{array}\right)+K^{-1} K^{-1}\left(\begin{array}{cccc}
1 & 0 & 0 & 0 \\
0 & 0 & -\omega_{3} & \omega_{2} \\
0 & \omega_{3} & 0 & -\omega_{1} \\
0 & -\omega_{2} & \omega_{1} & 0
\end{array}\right)\left(\begin{array}{c}
0 \\
\xi \\
\eta \\
\zeta
\end{array}\right)
\end{aligned}
$$

The coordinate system $O_{n} \overline{i_{1} i_{2}} \bar{i}_{3}$ rotates relative to the coordinate system $O_{n} \bar{I}_{1} \bar{I}_{2} \bar{I}_{3}$ with an angular velocity $\bar{\omega}$, which can be equal to zero. The coordinate system $O_{n} \bar{I}_{1} \bar{I}_{2} \bar{I}_{3}$ rotates relative to the coordinate system $O_{n} \overline{i_{1}} \bar{i}_{2} \bar{i}_{3}$ with angular velocity $-\bar{\omega}$.

\section{CALCULATION OF LAGRANGE FUNCTION}

Lagrange function for the relativistic rotation of the rigid body is derived from Lagrange function of the non-rotating point of masses system in the relativistic approximation, which has a form (Landau, Lifshitz, 1967):

$$
\begin{aligned}
L & =\frac{1}{2} \sum_{i} m_{i} \dot{\bar{R}}_{i}^{2}+\frac{1}{2} \sum_{i} \sum_{k \neq i} \frac{G m_{i} m_{k}}{\Delta_{i k}}+\frac{1}{c^{2}}\left\{\frac{3}{2} \sum_{i} \sum_{k \neq i} \frac{G m_{i} m_{k}}{\Delta_{i k}} \dot{\bar{R}}_{i}^{2}+\frac{1}{8} \sum_{i} m_{i} \dot{\bar{R}}_{i}^{4}-\right. \\
& \left.-\frac{1}{4} \sum_{i} \sum_{k \neq i} \frac{G m_{i} m_{k}}{\Delta_{i k}}\left[7 \dot{\bar{R}}_{i} \cdot \dot{\bar{R}}_{k}+\dot{\bar{R}}_{i} \cdot \frac{\bar{R}_{i}-\bar{R}_{k}}{\Delta_{i k}} \dot{\bar{R}}_{k} \cdot \frac{\bar{R}_{i}-\bar{R}_{k}}{\Delta_{i k}}+2 \sum_{s \neq i} \frac{G m_{s}}{\Delta_{i s}}\right]\right\} .
\end{aligned}
$$

Here $m_{i}, m_{k}, m_{s}$ are the mass of the $i$-th, $k$-th and $\mathrm{s}$-th points, respectively; $\Delta_{i j}=\sqrt{\left(X_{i}-X_{j}\right)^{2}+\left(Y_{i}-Y_{j}\right)^{2}+\left(Z_{i}-Z_{j}\right)^{2}}=\left|\bar{R}_{i}-\bar{R}_{j}\right|$, where $j=k$ or $s ; \quad \bar{R}_{i}, \dot{\bar{R}}_{i}, \bar{R}_{k}, \dot{\bar{R}}_{k} \quad$ are barycentric positions and velocities of these points of mass; c is velocity of light in vacuum; $G$ is the gravitational constant.

In the following decompositions of the sums, only in body terms for the single sum and perturbing terms between masses of the rigid body and others point of masses in the second sums are retained: 


$$
\begin{array}{l|l}
\sum_{i} f_{i}=\sum_{n} f_{n}+\sum_{j} f_{j}= & \sum_{i} \sum_{l \neq i} f_{i l}=\sum_{n} \sum_{j} f_{n j}+\sum_{j} \sum_{k \neq j} f_{j k}+\sum_{j} \sum_{n} f_{j n}= \\
=\sum_{n} f_{n}+\sum_{j} f_{j} & =\sum_{n} \sum_{j} f_{n j}+\sum_{j \neq j} f_{j k}+\sum_{j} \sum_{n} f_{j n} \\
\begin{array}{l}
\text { in body out body } \\
\text { terms terms }
\end{array} & \begin{array}{l}
\text { pertubations out body pertubations } \\
\text { mixed terms terms mixed terms }
\end{array} \\
\Rightarrow \sum_{i} \sum_{l \neq i} f_{i l} \sum_{s \neq i} g_{i s}=\sum_{n} \sum_{j} f_{n j} \sum_{j} g_{n j}+\sum_{j} \sum_{n} f_{j n} \sum_{n} g_{j n}
\end{array}
$$

Here $f_{i}, f_{i l}, g_{\text {is }}$ are some functions of point bodies under sums of the expression (2.1).

$$
\begin{aligned}
& L=\frac{1}{2} \sum_{n} d m_{n} \dot{\bar{R}}_{n}^{* 2}+\frac{1}{2} \sum_{j} m_{j} \dot{\bar{R}}_{j}^{2}+\frac{1}{2} \sum_{n} \sum_{j} \frac{G d m_{n} m_{j}}{\Delta_{n j}^{*}}+\frac{1}{2} \sum_{j} \sum_{k \neq j} \frac{G m_{j} m_{k}}{\Delta_{j k}}+\frac{1}{2} \sum_{j} \sum_{n} \frac{G m_{j} d m_{n}}{\Delta_{n j}^{*}}+ \\
& +\frac{1}{c^{2}}\left\{\frac{3}{2} \sum_{n} \sum_{j} \frac{G d m_{n} m_{j}}{\Delta_{n j}^{*}} \dot{\bar{R}}_{n}^{* 2}+\frac{3}{2} \sum_{j} \sum_{k \neq j} \frac{G m_{j} m_{k}}{\Delta_{j k}} \dot{\bar{R}}_{j}^{2}+\frac{3}{2} \sum_{j} \sum_{n} \frac{G m_{j} d m_{n}}{\Delta_{n j}^{*}} \dot{\bar{R}}_{j}^{2}+\right. \\
& +\frac{1}{8} \sum_{n} d m_{n} \dot{\bar{R}}_{n}^{* 4}+\frac{1}{8} \sum_{j} m_{j} \dot{\bar{R}}_{j}^{4}- \\
& -\frac{1}{4} \sum_{n} \sum_{j} \frac{G d m_{n} m_{j}}{\Delta_{n j}^{*}}\left[7 \dot{\bar{R}}_{n}^{*} \cdot \dot{\bar{R}}_{j}+\dot{\bar{R}}_{n}^{*} \cdot \frac{\bar{R}_{n}^{*}-\bar{R}_{j}}{\Delta_{n j}^{*}} \dot{\bar{R}}_{j} \cdot \frac{\bar{R}_{n}^{*}-\bar{R}_{j}}{\Delta_{n j}^{*}}+2 \sum_{j} \frac{G m_{j}}{\Delta_{n j}^{*}}\right]- \\
& \left.-\frac{1}{4} \sum_{j} \sum_{n} \frac{G m_{j} d m_{n}}{\Delta_{n j}^{*}}\left[7 \dot{\bar{R}}_{j} \cdot \dot{\bar{R}}_{n}^{*}+\dot{\bar{R}}_{j} \cdot \frac{\bar{R}_{j}-\bar{R}_{n}^{*}}{\Delta_{n j}^{*}} \dot{\bar{R}}_{n}^{*} \cdot \frac{\bar{R}_{j}-\bar{R}_{n}^{*}}{\Delta_{n j}^{*}}+2 \sum_{n} \frac{G d m_{n}}{\Delta_{n j}^{*}}\right]\right\}=
\end{aligned}
$$

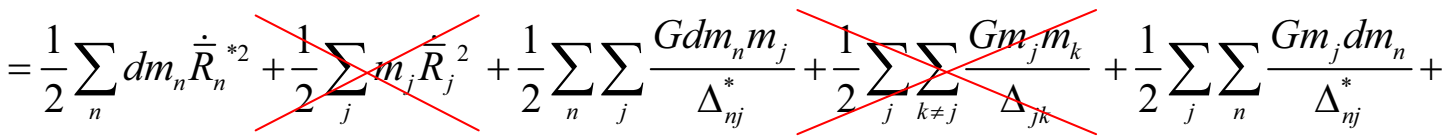

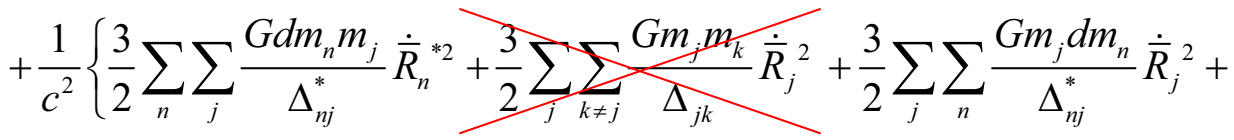

$$
\begin{aligned}
& +\frac{1}{8} \sum_{n} d m_{n} \dot{\bar{R}}_{n}^{* 4}+\frac{1}{8} \sum_{j}{ }_{1} \hat{R}_{j}^{4}- \\
& -\frac{1}{4} \sum_{n} \sum_{j} \frac{G d m_{n} m_{j}}{\Delta_{n j}^{*}}\left[7 \dot{\bar{R}}_{n}^{*} \cdot \dot{\bar{R}}_{j}+\dot{\bar{R}}_{n}^{*} \cdot \frac{\bar{R}_{n}^{*}-\bar{R}_{j}}{\Delta_{n j}^{*}} \dot{\bar{R}}_{j} \cdot \frac{\bar{R}_{n}^{*}-\bar{R}_{j}}{\Delta_{n j}^{*}}+2 \sum_{j} \frac{G m_{j}}{\Delta_{n j}^{*}}\right]- \\
& \left.-\frac{1}{4} \sum_{j} \sum_{n} \frac{G m_{j} d m_{n}}{\Delta_{n j}^{*}}\left[7 \dot{\bar{R}}_{j} \cdot \dot{\bar{R}}_{n}^{*}+\dot{\bar{R}}_{j} \cdot \frac{\bar{R}_{j}-\bar{R}_{n}^{*}}{\Delta_{n j}^{*}} \dot{\bar{R}}_{n}^{*} \cdot \frac{\bar{R}_{j}-\bar{R}_{n}^{*}}{\Delta_{n j}^{*}}+2 \sum_{n} \frac{G d m_{n}}{\Delta_{n j}^{*}}\right]\right\}
\end{aligned}
$$

After the reduction of similar terms Lagrange function for the relativistic rotation of the rigid body $m_{n}$ has the form: 


$$
\begin{aligned}
L_{n}= & \frac{1}{2} \sum_{n} d m_{n} \dot{\bar{R}}_{n}^{* 2}+\sum_{n} \sum_{j} \frac{G d m_{n} m_{j}}{\Delta_{n j}^{*}}+ \\
+ & \frac{1}{c^{2}}\left\{\frac{3}{2} \sum_{n} \sum_{j} \frac{G d m_{n} m_{j}}{\Delta_{n j}^{*}} \dot{\bar{R}}_{n}^{* 2}+\frac{3}{2} \sum_{n} \sum_{j} \frac{G d m_{n} m_{j}}{\Delta_{n j}^{*}} \dot{\bar{R}}_{j}^{2}+\frac{1}{8} \sum_{n} d m_{n} \dot{\bar{R}}_{n}^{* 4}-\right. \\
& -\frac{1}{2} \sum_{n} \sum_{j} \frac{G d m_{n} m_{j}}{\Delta_{n j}^{*}}\left[7 \dot{\bar{R}}_{n}^{*} \cdot \dot{\bar{R}}_{j}+\dot{\bar{R}}_{n}^{*} \cdot \frac{\bar{R}_{n}^{*}-\bar{R}_{j}}{\Delta_{n j}^{*}} \dot{\bar{R}}_{j} \cdot \frac{\bar{R}_{n}^{*}-\bar{R}_{j}}{\Delta_{n j}^{*}}\right]- \\
& \left.-\frac{1}{2} \sum_{n} d m_{n}\left(\sum_{j} \frac{G m_{j}}{\Delta_{n j}^{*}}\right)^{2}-\frac{1}{2} \sum_{j} m_{j}\left(\sum_{n} \frac{G d m_{n}}{\Delta_{n j}^{*}}\right)^{2}\right\}
\end{aligned}
$$

After the easy replacement of the summation signs with respect to the index $n$ by the integration signs over the range $m_{n}$ these terms take the form:

$$
\begin{aligned}
L_{n}= & \frac{1}{2} \int_{m_{n}} \dot{\bar{R}}_{n}^{* 2} d m_{n}+\sum_{j \neq n} G m_{j} \int_{m_{n}} \frac{d m_{n}}{\Delta_{n j}^{*}}+ \\
& +\frac{1}{8 c^{2}} \int_{m_{n}} \dot{\bar{R}}_{n}^{* 4} d m_{n}+\sum_{j \neq n} \frac{G m_{j}}{c^{2}}\left\{\frac{3}{2} \int_{m_{n}} \dot{\bar{R}}_{n}^{* 2} \frac{d m_{n}}{\Delta_{n j}^{*}}+\frac{3}{2} \dot{\bar{R}}_{j}^{2} \int_{m_{n}} \frac{d m_{n}}{\Delta_{n j}^{*}}-\right. \\
& \left.-\frac{7}{2} \int_{m_{n}} \dot{\bar{R}}_{n}^{*} \cdot \dot{\bar{R}}_{j} \frac{d m_{n}}{\Delta_{n j}^{*}}-\frac{1}{2} \int_{m_{n}} \dot{\bar{R}}_{n}^{*} \cdot \frac{\left(\bar{R}_{n}^{*}-\bar{R}_{j}\right)}{\Delta_{n j}^{*}} \dot{\bar{R}}_{j} \cdot \frac{\left(\bar{R}_{n}^{*}-\bar{R}_{j}\right)}{\Delta_{n j}^{*}} \frac{d m_{n}}{\Delta_{n j}^{*}}\right\}- \\
- & \frac{1}{2 c^{2}} \int_{m_{n}}\left(\sum_{j \neq n} \frac{G m_{j}}{\Delta_{n j}^{*}}\right)^{2} d m_{n}-\frac{1}{2 c^{2}} \sum_{j \neq n} m_{j}\left(\int_{m_{n}} \frac{G d m_{n}}{\Delta_{n j}^{*}}\right)^{2} \cdot
\end{aligned}
$$

Here and further $\Delta_{n j}^{*}=\left|\bar{R}_{n}-\bar{R}_{j}+\bar{\rho}\right|$. Usually in celestial mechanics $\bar{\rho}<<\bar{\Delta}_{n j}$. The integrands are expanded in Taylor series in powers of the parameter $\frac{|\bar{\rho}|}{\Delta_{n j}}$, which is a small quantity, for example, in view of the fact that the dimensions of the large bodies of the solar system are small in comparison with the distances between them. By the definition of the coordinate system $O_{n} \overline{i_{1}} \dot{i}_{2} \bar{i}_{3}$ all integrals of the form

$\int_{m_{n}} \xi d m_{n}, \int_{m_{n}} \eta d m_{n}, \int_{m_{n}} \zeta d m_{n}, \int_{m_{n}} \xi \eta d m_{n}, \int_{m_{n}} \eta \zeta d m_{n}, \int_{m_{n}} \zeta \xi d m_{n} \quad$ are identically equal to zero (2.4) (MacMillan, 1936).

In order to calculate certain integrals in Lagrange function (2.3), it is convenient to have some formulas and expressions, while using the identical transformations of the mixed product of vectors $\bar{a} \cdot(\bar{b} \times \bar{c})=\bar{b} \cdot(\bar{c} \times \bar{a})=\bar{c} \cdot(\bar{a} \times \bar{b})$ :

$\dot{\bar{R}}_{n}^{* 2}=\dot{\bar{R}}_{n}^{2}+2 \dot{\bar{R}}_{n} \cdot(\bar{\omega} \times \bar{\rho})+(\bar{\omega} \times \bar{\rho})^{2}=\dot{\bar{R}}_{n}^{2}+2 \bar{\rho} \cdot\left(\dot{\bar{R}}_{n} \times \bar{\omega}\right)+(\bar{\omega} \times \bar{\rho})^{2} ;$

$\dot{\bar{R}}_{n}^{*} \cdot \dot{\bar{R}}_{j}=\dot{\bar{R}}_{n} \cdot \dot{\bar{R}}_{j}+\dot{\bar{R}}_{j} \cdot(\bar{\omega} \times \bar{\rho})=\dot{\bar{R}}_{n} \cdot \dot{\bar{R}}_{j}+\bar{\rho} \cdot\left(\dot{\bar{R}}_{j} \times \bar{\omega}\right)$;

$\Delta_{n j}^{*}=\sqrt{\Delta_{n j}^{2}+2 \bar{\rho} \cdot\left(\bar{R}_{n}-\bar{R}_{j}\right)+\bar{\rho}^{2}} \Rightarrow$ 


$$
\begin{aligned}
& \frac{1}{\Delta_{n j}^{*}}=\frac{1}{\Delta_{n j}}\left[1+2 \frac{\bar{\rho} \cdot\left(\bar{R}_{n}-\bar{R}_{j}\right)}{\Delta_{n j}^{2}}+\frac{\bar{\rho}^{2}}{\Delta_{n j}^{2}}\right]^{-\frac{1}{2}}=\frac{1}{\Delta_{n j}}-\frac{\bar{\rho} \cdot\left(\bar{R}_{n}-\bar{R}_{j}\right)}{\Delta_{n j}^{3}}-\frac{\bar{\rho}^{2}}{2 \Delta_{n j}^{3}}+\frac{3}{2} \frac{\left[\bar{\rho} \cdot\left(\bar{R}_{n}-\bar{R}_{j}\right)\right]^{2}}{\Delta_{n j}^{5}}+\ldots ; \\
& \frac{1}{\Delta_{n j}^{* 2}}=\frac{1}{\Delta_{n j}^{2}}\left[1+2 \frac{\bar{\rho} \cdot\left(\bar{R}_{n}-\bar{R}_{j}\right)}{\Delta_{n j}^{2}}+\frac{\bar{\rho}^{2}}{\Delta_{n j}^{2}}\right]^{-2}=\frac{1}{\Delta_{n j}^{2}}\left\{1-4 \frac{\bar{\rho} \cdot\left(\bar{R}_{n}-\bar{R}_{j}\right)}{\Delta_{n j}^{2}}-2 \frac{\bar{\rho}^{2}}{\Delta_{n j}^{2}}+12 \frac{\left[\bar{\rho} \cdot\left(\bar{R}_{n}-\bar{R}_{j}\right)\right]^{2}}{\Delta_{n j}^{4}}+\ldots\right\} ; \\
& \frac{1}{\Delta_{n j}^{* 3}}=\frac{1}{\Delta_{n j}^{3}}\left[1+2 \frac{\bar{\rho} \cdot\left(\bar{R}_{n}-\bar{R}_{j}\right)}{\Delta_{n j}^{2}}+\frac{\bar{\rho}^{2}}{\Delta_{n j}^{2}}\right]^{-\frac{3}{2}}=\frac{1}{\Delta_{n j}^{3}}-3 \frac{\bar{\rho} \cdot\left(\bar{R}_{n}-\bar{R}_{j}\right)}{\Delta_{n j}^{5}}-\frac{3}{2} \frac{\bar{\rho}^{2}}{\Delta_{n j}^{5}}+\frac{15}{2} \frac{\left[\bar{\rho} \cdot\left(\bar{R}_{n}-\bar{R}_{j}\right)\right]^{2}}{\Delta_{n j}^{7}}+\ldots ;
\end{aligned}
$$

Analytical calculations of auxiliary formulas, expressions and integrals necessary for calculating the Lagrange function (2.3) for the relativistic rotation of the rigid body $m_{n}$ are given in Appendix A.

\section{1 Calculation of the Newtonian part of the Lagrange function}

The first line of the expression (2.3) turned out to be the Newtonian part of Lagrange function for the relativistic rotation of the rigid body:

$$
L_{n}^{\text {Newton }}=\frac{1}{2} \int_{m_{n}} \dot{\bar{R}}_{n}^{* 2} d m_{n}+\sum_{j \neq n} G m_{j} \int_{m_{n}} \frac{d m_{n}}{\Delta_{n j}^{*}}
$$

The first certain integral, which is dependent from $\dot{\bar{R}}_{n}^{*}$, is computed by using integral (A.1):

$$
\frac{1}{2} \int_{m_{n}} \dot{\bar{R}}_{n}^{* 2} d m_{n}=\frac{1}{2} \dot{\bar{R}}_{n}^{2} m_{n}+\frac{1}{2} \int_{m_{n}}(\bar{\omega} \times \bar{\rho})^{2} d m_{n}=\frac{1}{2} \dot{\bar{R}}_{n}^{2} m_{n}+\frac{1}{2} \bar{H}_{n} \cdot \bar{\omega} \text { is the kinetic energy of the }
$$

body $m_{n}$ (the sum of the kinetic energy of the translational motion and of the rotational motion). The second certain integral, which is independent from $\dot{\bar{R}}_{n}^{*}$, is computed by using integrals (A.2) and (A.3):

$$
\begin{aligned}
& \sum_{j \neq n} G m_{j} \int_{m_{n}} \frac{d m_{n}}{\Delta_{n j}^{*}}=\sum_{j \neq n} G m_{j}\left\langle\frac{m_{n}}{\Delta_{n j}}-\frac{1}{2 \Delta_{n j}^{3}} \int_{m_{n}} \bar{\rho}^{2} d m_{n}+\frac{3}{2 \Delta_{n j}^{5}} \int_{m_{n}}\left[\bar{\rho} \cdot\left(\bar{R}_{n}-\bar{R}_{j}\right)\right]^{2} d m_{n}\right\rangle= \\
& =\sum_{j \neq n} G m_{j}\left\langle\frac{m_{n}}{\Delta_{n j}}+\right. \\
& +\frac{1}{2 \Delta_{n j}^{3}}\left\{A_{n}+B_{n}+C_{n}-\frac{3}{\Delta_{n j}^{2}}\left[A_{n}\left(x_{n}-x_{j}\right)^{2}+B_{n}\left(y_{n}-y_{j}\right)^{2}+C_{n}\left(z_{n}-z_{j}\right)^{2}\right]\right\} ;
\end{aligned}
$$

It is the force function of gravitational interaction of the body $m_{n}$ with other bodies.

\section{2 Calculation of the relativistic part of the Lagrange function}

The relativistic part of the Lagrange function for the relativistic rotation of the rigid body, taking into account the interaction of the body $m_{n}$ with other bodies, can be represented as follows: 


$$
\begin{aligned}
\Delta L_{n} & =\frac{1}{8 c^{2}} \int_{m_{n}} \dot{\bar{R}}_{n}^{* 4} d m_{n}+\sum_{j \neq n} \frac{G m_{j}}{c^{2}}\left\{\frac{3}{2} \int_{m_{n}} \dot{\bar{R}}_{n}^{* 2} \frac{d m_{n}}{\Delta_{n j}^{*}}+\frac{3}{2} \dot{\bar{R}}_{j}^{2} \int_{m_{n}} \frac{d m_{n}}{\Delta_{n j}^{*}}-\frac{7}{2} \int_{m_{n}} \dot{\bar{R}}_{n}^{*} \cdot \dot{\bar{R}}_{j} \frac{d m_{n}}{\Delta_{n j}^{*}}-\right. \\
& \left.-\frac{1}{2} \int_{m_{n}} \dot{\bar{R}}_{n}^{*} \cdot \frac{\left(\bar{R}_{n}^{*}-\bar{R}_{j}\right)}{\Delta_{n j}^{*}} \dot{\bar{R}}_{j} \cdot \frac{\left(\bar{R}_{n}^{*}-\bar{R}_{j}\right)}{\Delta_{n j}^{*}} \frac{d m_{n}}{\Delta_{n j}^{*}}\right\}- \\
& -\frac{1}{2 c^{2}} \int_{m_{n}}\left(\sum_{j \neq n} \frac{G m_{j}}{\Delta_{n j}^{*}}\right)^{2} d m_{n}-\frac{1}{2 c^{2}} \sum_{j \neq n} m_{j}\left(\int_{m_{n}} \frac{G d m_{n}}{\Delta_{n j}^{*}}\right)^{2} .
\end{aligned}
$$

\section{2.1 Calculation of definite integrals dependent on $\dot{\bar{R}}_{n}^{*}$}

The geodesic rotation of the set of point masses forming the body $m_{n}$ is generated by the relativistic terms of the Lagrange function $\Delta L_{n}$, which contain the angular velocity of rotation of the body $m_{n}$ around its own center of mass $\bar{\omega}$, that is depending on the barycentric velocity vector $\dot{\bar{R}}_{n}^{*}$ of the element $d m_{n}$ :

$$
\begin{aligned}
\Delta L_{n}^{G} & =\frac{1}{8 c^{2}} \int_{m_{n}} \dot{\bar{R}}_{n}^{* 4} d m_{n}+\sum_{j \neq n} \frac{G m_{j}}{c^{2}}\left\{\frac{3}{2} \int_{m_{n}} \dot{\bar{R}}_{n}^{* 2} \frac{d m_{n}}{\Delta_{n j}^{*}}-\frac{7}{2} \int_{m_{n}} \dot{\bar{R}}_{n}^{*} \cdot \dot{\bar{R}}_{j} \frac{d m_{n}}{\Delta_{n j}^{*}}-\right. \\
& \left.-\frac{1}{2} \int_{m_{n}} \dot{\bar{R}}_{n}^{*} \cdot \frac{\left(\bar{R}_{n}^{*}-\bar{R}_{j}\right)}{\Delta_{n j}^{*}} \dot{\bar{R}}_{j} \cdot \frac{\left(\bar{R}_{n}^{*}-\bar{R}_{j}\right)}{\Delta_{n j}^{*}} \frac{d m_{n}}{\Delta_{n j}^{*}}\right\} .
\end{aligned}
$$

As a result of combining integrals (A.4) and (A.1), the integral $\frac{1}{8} \int_{m_{n}} \dot{\bar{R}}_{n}^{* 4} d m_{n}$ is computed:

$$
\begin{aligned}
\frac{1}{8} \int_{m_{n}} \dot{\bar{R}}_{n}^{* 4} d m_{n}= & \frac{1}{8} \dot{\bar{R}}_{n}^{4} m_{n}+\frac{1}{2} \int_{m_{n}}\left[\bar{\rho} \cdot\left(\dot{\bar{R}}_{n} \times \bar{\omega}\right)\right]^{2} d m_{n}+\frac{1}{4} \dot{\bar{R}}_{n}^{2} \int_{m_{n}}(\bar{\omega} \times \bar{\rho})^{2} d m_{n}= \\
= & \frac{1}{8} \dot{\bar{R}}_{n}^{4} m_{n}+\frac{1}{4} \dot{\bar{R}}_{n}^{2}\left(A_{n} \omega_{1}^{2}+B_{n} \omega_{2}^{2}+C_{n} \omega_{3}^{2}\right)+\left(\dot{y}_{n} \omega_{3}-\dot{z}_{n} \omega_{2}\right)^{2} \frac{B_{n}+C_{n}-A_{n}}{4}+ \\
& +\left(\dot{z}_{n} \omega_{1}-\dot{x}_{n} \omega_{3}\right)^{2} \frac{C_{n}+A_{n}-B_{n}}{4}+\left(\dot{x}_{n} \omega_{2}-\dot{y}_{n} \omega_{1}\right)^{2} \frac{A_{n}+B_{n}-C_{n}}{4}
\end{aligned}
$$

As a result of combining integrals (A.1), (A.5), (A.2) and (A.3), the integral $\frac{3}{2} \int_{m_{n}} \frac{\dot{\bar{R}}_{n}^{* 2}}{\Delta_{n j}^{*}} d m_{n}$ is computed:

$$
\begin{aligned}
& \frac{3}{2} \int_{m_{n}} \frac{\dot{\bar{R}}_{n}^{* 2}}{\Delta_{n j}^{*}} d m_{n}=\frac{3}{2} \frac{\dot{\bar{R}}_{n}^{2}}{\Delta_{n j}} m_{n}+\frac{3}{2 \Delta_{n j}} \int_{m_{n}}(\bar{\omega} \times \bar{\rho})^{2} d m_{n}-\frac{3}{\Delta_{n j}^{3}} \int_{m_{n}} \bar{\rho} \cdot\left(\dot{\bar{R}}_{n} \times \bar{\omega}\right) \bar{\rho} \cdot\left(\bar{R}_{n}-\bar{R}_{j}\right) d m_{n}- \\
& \quad-\frac{3 \dot{\bar{R}}_{n}^{2}}{4 \Delta_{n j}^{3}} \int_{m_{n}} \bar{\rho}^{2} d m_{n}+\frac{9}{4} \frac{\dot{\bar{R}}_{n}^{2}}{\Delta_{n j}^{5}} \int_{m_{n}}\left[\bar{\rho} \cdot\left(\bar{R}_{n}-\bar{R}_{j}\right)\right]^{2} d m_{n}=\frac{3}{2} \frac{\dot{\bar{R}}_{n}^{2}}{\Delta_{n j}} m_{n}+\frac{3 \dot{\bar{R}}_{n}^{2}}{4 \Delta_{n j}^{3}}\left\{A_{n}+B_{n}+C_{n}-\right. \\
& \left.\quad-\frac{3}{\Delta_{n j}^{2}}\left[A_{n}\left(x_{n}-x_{j}\right)^{2}+B_{n}\left(y_{n}-y_{j}\right)^{2}+C_{n}\left(z_{n}-z_{j}\right)^{2}\right]\right\}-\frac{3}{2 \Delta_{n j}^{3}}\left\{\bar{H}_{n} \cdot\left(\bar{R}_{n}-\bar{R}_{j}\right) \times \dot{\bar{R}}_{n}+\right. \\
& +C_{n}\left(z_{n}-z_{j}\right)\left(\omega_{1} \dot{y}_{n}-\omega_{2} \dot{x}_{n}\right)+B_{n}\left(y_{n}-y_{j}\right)\left(\omega_{3} \dot{x}_{n}-\omega_{1} \dot{z}_{n}\right)+A_{n}\left(x_{n}-x_{j}\right)\left(\omega_{2} \dot{z}_{n}-\omega_{3} \dot{y}_{n}\right)+ \\
& +C_{n} \dot{z}_{n}\left[\omega_{1}\left(y_{n}-y_{j}\right)-\omega_{2}\left(x_{n}-x_{j}\right)\right]+B_{n} \dot{y}_{n}\left[\omega_{3}\left(x_{n}-x_{j}\right)-\omega_{1}\left(z_{n}-z_{j}\right)\right]+
\end{aligned}
$$




$$
\left.+A_{n} \dot{x}_{n}\left[\omega_{2}\left(z_{n}-z_{j}\right)-\omega_{3}\left(y_{n}-y_{j}\right)\right]\right\}+\frac{3}{2 \Delta_{n j}}\left(A_{n} \omega_{1}^{2}+B_{n} \omega_{2}^{2}+C_{n} \omega_{3}^{2}\right)
$$

As a result of combining integrals (A.6), (A.2) and (A.3), the integral $-\frac{7}{2} \int_{m_{n}} \frac{\dot{\bar{R}}_{n}^{*} \cdot \dot{\bar{R}}_{j}}{\Delta_{n j}^{*}} d m_{n}$ is computed:

$$
\begin{aligned}
& -\frac{7}{2} \int_{m_{n}} \frac{\dot{\bar{R}}_{n}^{*} \cdot \dot{\bar{R}}_{j}}{\Delta_{n j}^{*}} d m_{n}=-\frac{7}{2} \frac{\dot{\bar{R}}_{n} \cdot \dot{\bar{R}}_{j}}{\Delta_{n j}} m_{n}+\frac{7}{2 \Delta_{n j}^{3}} \int_{m_{n}} \bar{\rho} \cdot\left(\dot{\bar{R}}_{j} \times \bar{\omega}\right) \bar{\rho} \cdot\left(\bar{R}_{n}-\bar{R}_{j}\right) d m_{n}+\frac{7 \dot{\bar{R}}_{n} \cdot \dot{\bar{R}}_{j}}{4 \Delta_{n j}^{3}} \int_{m_{n}} \bar{\rho}^{2} d m_{n}- \\
& -\frac{21}{4} \frac{\dot{\bar{R}}_{n} \cdot \dot{\bar{R}}_{j}}{\Delta_{n j}^{5}} \int\left[m_{n}\left[\bar{\rho} \cdot\left(\bar{R}_{n}-\bar{R}_{j}\right)\right]^{2} d m_{n}=-\frac{7}{2} \frac{\dot{\bar{R}}_{n} \cdot \dot{\bar{R}}_{j}}{\Delta_{n j}} m_{n}-\frac{7}{4} \frac{\dot{\bar{R}}_{n} \cdot \dot{\bar{R}}_{j}}{\Delta_{n j}^{3}}\left\{A_{n}+B_{n}+C_{n}-\right.\right. \\
& \left.-\frac{3}{\Delta_{n j}^{2}}\left[A_{n}\left(x_{n}-x_{j}\right)^{2}+B_{n}\left(y_{n}-y_{j}\right)^{2}+C_{n}\left(z_{n}-z_{j}\right)^{2}\right]\right\}+\frac{7}{4 \Delta_{n j}^{3}}\left\{\bar{H}_{n} \cdot\left(\bar{R}_{n}-\bar{R}_{j}\right) \times \dot{\bar{R}}_{j}+\right. \\
& +C_{n}\left(z_{n}-z_{j}\right)\left(\omega_{1} \dot{y}_{n}-\omega_{2} \dot{x}_{n}\right)+B_{n}\left(y_{n}-y_{j}\right)\left(\omega_{3} \dot{x}_{n}-\omega_{1} \dot{z}_{n}\right)+A_{n}\left(x_{n}-x_{j}\right)\left(\omega_{2} \dot{z}_{n}-\omega_{3} \dot{y}_{n}\right)+ \\
& +C_{n} \dot{z}_{n}\left[\omega_{1}\left(y_{n}-y_{j}\right)-\omega_{2}\left(x_{n}-x_{j}\right)\right]+B_{n} \dot{y}_{n}\left[\omega_{3}\left(x_{n}-x_{j}\right)-\omega_{1}\left(z_{n}-z_{j}\right)\right]+ \\
& \left.+A_{n} \dot{x}_{n}\left[\omega_{2}\left(z_{n}-z_{j}\right)-\omega_{3}\left(y_{n}-y_{j}\right)\right]\right\} \text {; }
\end{aligned}
$$

As a result of combining integrals (A.2), (A.3), (A.7), (A.8), (A.9), (A.10) and (A.12), next integral is computed:

$$
\begin{aligned}
& -\frac{1}{2} \int_{m_{n}} \dot{\bar{R}}_{n}^{*} \cdot \frac{\left(\bar{R}_{n}^{*}-\bar{R}_{j}\right)}{\Delta_{n j}^{*}} \dot{\bar{R}}_{j} \cdot \frac{\left(\bar{R}_{n}^{*}-\bar{R}_{j}\right)}{\Delta_{n j}^{*}} \frac{1}{\Delta_{n j}^{*}} d m_{n}=-\dot{\bar{R}}_{n} \cdot\left(\bar{R}_{n}-\bar{R}_{j}\right) \dot{\bar{R}}_{j} \cdot\left(\bar{R}_{n}-\bar{R}_{j}\right) \frac{1}{2 \Delta_{n j}^{3} \int_{m_{n}}} d m_{n}+ \\
& +\dot{\bar{R}}_{n} \cdot\left(\bar{R}_{n}-\bar{R}_{j}\right) \dot{\bar{R}}_{j} \cdot\left(\bar{R}_{n}-\bar{R}_{j}\right) \frac{3}{4 \Delta_{n j}^{5}} \int_{m_{n}} \bar{\rho}^{2} d m_{n}-\dot{\bar{R}}_{n} \cdot\left(\bar{R}_{n}-\bar{R}_{j}\right) \dot{\bar{R}}_{j} \cdot\left(\bar{R}_{n}-\bar{R}_{j}\right) \frac{15}{4 \Delta_{n j}^{7}} \int\left[m_{n}\left[\bar{\rho} \cdot\left(\bar{R}_{n}-\bar{R}_{j}\right)\right]^{2} d m_{n}+\right. \\
& +\frac{3 \dot{\bar{R}}_{n} \cdot\left(\bar{R}_{n}-\bar{R}_{j}\right)}{2 \Delta_{n j}^{5}} \int_{m_{n}} \bar{\rho} \cdot \dot{\bar{R}} \bar{\rho} \cdot\left(\bar{R}_{n}-\bar{R}_{j}\right) d m_{n}+\frac{3 \dot{\bar{R}}_{j} \cdot\left(\bar{R}_{n}-\bar{R}_{j}\right)}{2 \Delta_{n j}^{5}} \int_{m_{n}} \bar{\rho} \cdot \dot{\bar{R}} \bar{\rho} \bar{\rho} \cdot\left(\bar{R}_{n}-\bar{R}_{j}\right) d m_{n}- \\
& -\frac{1}{2 \Delta_{n j}^{3}} \int_{m_{n}} \bar{\rho} \cdot \dot{R_{n}} \bar{\rho} \cdot \dot{\bar{R}}_{j} d m_{n}+\frac{3 \dot{\bar{R}}_{j} \cdot\left(\bar{R}_{n}-\bar{R}_{j}\right)}{2 \Delta_{n j}^{5}} \int_{m_{n}} \bar{\rho} \cdot\left[\left(\bar{R}_{n}-\bar{R}_{j}\right) \times \bar{\omega}\right] \bar{\rho} \cdot\left(\bar{R}_{n}-\bar{R}_{j}\right) d m_{n}- \\
& -\frac{1}{2 \Delta_{n j}^{3}} \int_{m_{n}} \bar{\rho} \cdot\left[\left(\bar{R}_{n}-\bar{R}_{j}\right) \times \bar{\omega}\right] \bar{\rho} \cdot \dot{\bar{R}}_{j} d m_{n}=-\frac{1}{2} \dot{\bar{R}}_{n} \cdot\left(\bar{R}_{n}-\bar{R}_{j}\right) \dot{\bar{R}}_{j} \cdot\left(\bar{R}_{n}-\bar{R}_{j}\right) \frac{1}{\Delta_{n j}^{3}} m_{n}+ \\
& +\dot{\bar{R}}_{n} \cdot\left(\bar{R}_{n}-\bar{R}_{j}\right) \dot{\bar{R}}_{j} \cdot\left(\bar{R}_{n}-\bar{R}_{j}\right) \frac{15}{4 \Delta_{n j}^{7}}\left[A_{n}\left(x_{n}-x_{j}\right)^{2}+B_{n}\left(y_{n}-y_{j}\right)^{2}+C_{n}\left(z_{n}-z_{j}\right)^{2}\right]- \\
& -\frac{3 \dot{\bar{R}}_{n} \cdot\left(\bar{R}_{n}-\bar{R}_{j}\right)}{2 \Delta_{n j}^{5}}\left[\dot{x}_{j}\left(x_{n}-x_{j}\right) A_{n}+\dot{y}_{j}\left(y_{n}-y_{j}\right) B_{n}+\dot{z}_{j}\left(z_{n}-z_{j}\right) C_{n}\right]- \\
& -\frac{3 \dot{\bar{R}}_{j} \cdot\left(\bar{R}_{n}-\bar{R}_{j}\right)}{2 \Delta_{n j}^{5}}\left[\dot{x}_{n}\left(x_{n}-x_{j}\right) A_{n}+\dot{y}_{n}\left(y_{n}-y_{j}\right) B_{n}+\dot{z}_{n}\left(z_{n}-z_{j}\right) C_{n}\right]- \\
& -\frac{\dot{\bar{R}}_{n} \cdot \dot{\bar{R}}_{j}}{4 \Delta_{n j}^{3}}\left(A_{n}+B_{n}+C_{n}\right)+\frac{1}{2 \Delta_{n j}^{3}}\left[\dot{x}_{n} \dot{x}_{j} A_{n}+\dot{y}_{n} \dot{y}_{j} B_{n}+\dot{z}_{n} \dot{z}_{j} C_{n}\right]+
\end{aligned}
$$




$$
\begin{aligned}
& +\frac{3 \dot{\bar{R}}_{j} \cdot\left(\bar{R}_{n}-\bar{R}_{j}\right)}{2 \Delta_{n j}^{5}}\left[\left(x_{n}-x_{j}\right)\left(y_{n}-y_{j}\right) \omega_{3}\left(B_{n}-A_{n}\right)+\left(z_{n}-z_{j}\right)\left(x_{n}-x_{j}\right) \omega_{2}\left(A_{n}-C_{n}\right)+\right. \\
& \left.+\left(y_{n}-y_{j}\right)\left(z_{n}-z_{j}\right) \omega_{1}\left(C_{n}-B_{n}\right)\right]+ \\
& +\frac{1}{4 \Delta_{n j}^{3}}\left\{\bar{H}_{n} \cdot\left(\bar{R}_{n}-\bar{R}_{j}\right) \times \dot{\bar{R}}_{j}-C_{n}\left(z_{n}-z_{j}\right)\left(\omega_{1} \dot{y}_{n}-\omega_{2} \dot{x}_{n}\right)-B_{n}\left(y_{n}-y_{j}\right)\left(\omega_{3} \dot{x}_{n}-\omega_{1} \dot{z}_{n}\right)-\right. \\
& -A_{n}\left(x_{n}-x_{j}\right)\left(\omega_{2} \dot{z}_{n}-\omega_{3} \dot{y}_{n}\right)-C_{n} \dot{z}_{n}\left[\omega_{1}\left(y_{n}-y_{j}\right)-\omega_{2}\left(x_{n}-x_{j}\right)\right]- \\
& \left.-B_{n} \dot{y}_{n}\left[\omega_{3}\left(x_{n}-x_{j}\right)-\omega_{1}\left(z_{n}-z_{j}\right)\right]-A_{n} \dot{x}_{n}\left[\omega_{2}\left(z_{n}-z_{j}\right)-\omega_{3}\left(y_{n}-y_{j}\right)\right]\right\} ;
\end{aligned}
$$

\section{2.2 Calculation of definite integrals independent of $\dot{\bar{R}}_{n}^{*}$}

$$
L_{n}^{N G}=\sum_{j \neq n} \frac{G m_{j}}{c^{2}} \frac{3}{2} \dot{\bar{R}}_{j}^{2} \int_{m_{n}} \frac{d m_{n}}{\Delta_{n j}^{*}}-\frac{1}{2 c^{2}} \int_{m_{n}}\left(\sum_{j \neq n} \frac{G m_{j}}{\Delta_{n j}^{*}}\right)^{2} d m_{n}-\frac{1}{2 c^{2}} \sum_{j \neq n} m_{j}\left(\int_{m_{n}} \frac{G d m_{n}}{\Delta_{n j}^{*}}\right)^{2} .
$$

As a result of combining integrals (A.2) and (A.3), the integral $\frac{3}{2} \dot{\bar{R}}_{j}^{2} \int_{m_{n}} \frac{d m_{n}}{\Delta_{n j}^{*}}$ is computed:

$\frac{3}{2} \dot{\bar{R}}_{j}^{2} \int_{m_{n}} \frac{d m_{n}}{\Delta_{n j}^{*}}=\frac{3}{2} \dot{\bar{R}}_{j}^{2} \frac{m_{n}}{\Delta_{n j}}-\frac{3 \dot{\bar{R}}_{j}^{2}}{4 \Delta_{n j}^{3}} \int_{m_{n}} \bar{\rho}^{2} d m_{n}+\frac{9 \dot{\bar{R}}_{j}^{2}}{4 \Delta_{n j}^{5}} \int_{m_{n}}\left[\bar{\rho} \cdot\left(\bar{R}_{n}-\bar{R}_{j}\right)\right]^{2} d m_{n}=$

$$
\begin{aligned}
& =\frac{3}{2} \dot{\bar{R}}_{j}^{2} \frac{m_{n}}{\Delta_{n j}}-\frac{3 \dot{\bar{R}}_{j}^{2}}{8 \Delta_{n j}^{3}}\left(A_{n}+B_{n}+C_{n}\right)+ \\
& +\frac{9 \dot{\bar{R}}_{j}^{2}}{8 \Delta_{n j}^{3}}\left\{A_{n}+B_{n}+C_{n}-\frac{2}{\Delta_{n j}^{2}}\left[A_{n}\left(x_{n}-x_{j}\right)^{2}+B_{n}\left(y_{n}-y_{j}\right)^{2}+C_{n}\left(z_{n}-z_{j}\right)^{2}\right]\right\}= \\
& =\frac{3}{2} \dot{\bar{R}}_{j}^{2} \frac{m_{n}}{\Delta_{n j}}+\frac{3 \dot{\bar{R}}_{j}^{2}}{4 \Delta_{n j}^{3}}\left\{A_{n}+B_{n}+C_{n}-\frac{3}{\Delta_{n j}^{2}}\left[A_{n}\left(x_{n}-x_{j}\right)^{2}+B_{n}\left(y_{n}-y_{j}\right)^{2}+C_{n}\left(z_{n}-z_{j}\right)^{2}\right]\right\} ;
\end{aligned}
$$

As a result of combining integrals (A.11), (A.2) and (A.3), computing the integral $-\frac{1}{2 c^{2}} \int_{m_{n}}\left(\sum_{j \neq n} \frac{G m_{j}}{\Delta_{n j}^{*}}\right)^{2} d m_{n}$ :

$$
\begin{aligned}
&-\frac{1}{2 c^{2}} \int_{m_{n}}\left(\sum_{j \neq n} \frac{G m_{j}}{\Delta_{n j}^{*}}\right)^{2} d m_{n}=-\frac{1}{2 c^{2}} \int_{m_{n}}\left(\sum_{j \neq n} \frac{G m_{j}}{\Delta_{n j}^{*}}\right)\left(\sum_{k \neq n} \frac{G m_{k}}{\Delta_{n k}^{*}}\right) d m_{n}= \\
&=-\frac{1}{2 c^{2}} \sum_{j \neq n} \sum_{k \neq n} G m_{j} G m_{k} \int_{m_{n}} \frac{1}{\Delta_{n j}^{*}} \frac{1}{\Delta_{n k}^{*}} d m_{n}= \\
&=-\frac{1}{2 c^{2}} \sum_{j \neq n} \sum_{k \neq n} \frac{G m_{j}}{\Delta_{n j}} \frac{G m_{k}}{\Delta_{n k}}\left\langle m_{n}+\frac{1}{\Delta_{n j}^{2}} \frac{1}{\Delta_{n k}^{2}} \int_{m_{n}} \bar{\rho} \cdot\left(\bar{R}_{n}-\bar{R}\right) \bar{\rho} \cdot\left(\bar{R}_{n}-\bar{R}_{k}\right) d m_{n}-\right. \\
&\left.-\frac{1}{2 \Delta_{n j}^{2}} \int_{m_{n}} \bar{\rho}^{2} d m_{n}+\frac{3}{2 \Delta_{n j}^{4}} \int_{m_{n}}\left[\bar{\rho} \cdot\left(\bar{R}_{n}-\bar{R}_{j}\right)\right]^{2} d m_{n}-\frac{1}{2 \Delta_{n k}^{2}} \int_{m_{n}} \bar{\rho}^{2} d m_{n}+\frac{3}{2 \Delta_{n k}^{4}} \int\left[\bar{\rho} \cdot\left(\bar{R}_{n}-\bar{R}_{k}\right)\right]^{2} d m_{n}\right\rangle=
\end{aligned}
$$




$$
\begin{array}{r}
=-\frac{1}{2 c^{2}} \sum_{j \neq n} \sum_{k \neq n} \frac{G m_{j}}{\Delta_{n j}} \frac{G m_{k}}{\Delta_{n k}}\left\{m_{n}+\frac{1}{2}\left[\frac{\left(\bar{R}_{n}-\bar{R}_{j}\right) \cdot\left(\bar{R}_{n}-\bar{R}_{k}\right)}{\Delta_{n j}^{2} \Delta_{n k}^{2}}+\frac{1}{2}\left(\frac{1}{\Delta_{n j}^{2}}+\frac{1}{\Delta_{n k}^{2}}\right)\right]\left(A_{n}+B_{n}+C_{n}\right)-\right. \\
-\frac{1}{\Delta_{n j}^{2} \Delta_{n k}^{2}}\left[A_{n}\left(x_{n}-x_{j}\right)\left(x_{n}-x_{k}\right)+B_{n}\left(y_{n}-y_{j}\right)\left(y_{n}-y_{k}\right)+C_{n}\left(z_{n}-z_{j}\right)\left(z_{n}-z_{k}\right)\right]- \\
-\frac{3}{2 \Delta_{n j}^{4}}\left[A_{n}\left(x_{n}-x_{j}\right)^{2}+B_{n}\left(y_{n}-y_{j}\right)^{2}+C_{n}\left(z_{n}-z_{j}\right)^{2}\right]- \\
\left.-\frac{3}{2 \Delta_{n k}^{4}}\left[A_{n}\left(x_{n}-x_{k}\right)^{2}+B_{n}\left(y_{n}-y_{k}\right)^{2}+C_{n}\left(z_{n}-z_{k}\right)^{2}\right]\right\} ;
\end{array}
$$

As it was obtained earlier

$$
\int_{m_{n}} \frac{d m_{n}}{\Delta_{n j}^{*}}=\frac{m_{n}}{\Delta_{n j}}+\frac{1}{2 \Delta_{n j}^{3}}\left\{A_{n}+B_{n}+C_{n}-\frac{3}{\Delta_{n j}^{2}}\left[A_{n}\left(x_{n}-x_{j}\right)^{2}+B_{n}\left(y_{n}-y_{j}\right)^{2}+C_{n}\left(z_{n}-z_{j}\right)^{2}\right]\right\} .
$$

Accordingly the integral $-\frac{1}{2 c^{2}} \sum_{j \neq n} m_{j}\left(\int_{m_{n}} \frac{G d m_{n}}{\Delta_{n j}^{*}}\right)^{2}$ is computed:

$$
\begin{aligned}
& -\frac{1}{2 c^{2}} \sum_{j \neq n} m_{j}\left(\int_{m_{n}} \frac{G d m_{n}}{\Delta_{n j}^{*}}\right)^{2}= \\
& =-\frac{1}{2 c^{2}} \sum_{j \neq n} m_{j} G^{2}\left\langle\frac{m_{n}}{\Delta_{n j}}+\frac{1}{2 \Delta_{n j}^{3}}\left\{A_{n}+B_{n}+C_{n}-\frac{3}{\Delta_{n j}^{2}}\left[A_{n}\left(x_{n}-x_{j}\right)^{2}+B_{n}\left(y_{n}-y_{j}\right)^{2}+C_{n}\left(z_{n}-z_{j}\right)^{2}\right]\right\}\right)^{2}= \\
& =-\frac{1}{2 c^{2}} \sum_{j \neq n} m_{j} G^{2}\left\langle\frac{m_{n}^{2}}{\Delta_{n j}^{2}}+\frac{m_{n}}{\Delta_{n j}^{4}}\left\{A_{n}+B_{n}+C_{n}-\frac{3}{\Delta_{n j}^{2}}\left[A_{n}\left(x_{n}-x_{j}\right)^{2}+B_{n}\left(y_{n}-y_{j}\right)^{2}+C_{n}\left(z_{n}-z_{j}\right)^{2}\right]\right\}+\ldots\right\rangle ;
\end{aligned}
$$

after omitted small terms $\leq o\left(\Delta_{n j}^{-6}\right)$ :

$$
\begin{aligned}
& -\frac{1}{2 c^{2}} \sum_{j \neq n} m_{j}\left(\int_{m_{n}} \frac{G d m_{n}}{\Delta_{n j}^{*}}\right)^{2}= \\
& =-\frac{G m_{n}}{2 c^{2}} \sum_{j \neq n} \frac{G m_{j}}{\Delta_{n j}^{2}}\left\langle m_{n}+\frac{1}{\Delta_{n j}^{2}}\left\{A_{n}+B_{n}+C_{n}-\frac{3}{\Delta_{n j}^{2}}\left[A_{n}\left(x_{n}-x_{j}\right)^{2}+B_{n}\left(y_{n}-y_{j}\right)^{2}+C_{n}\left(z_{n}-z_{j}\right)^{2}\right]\right\} .\right.
\end{aligned}
$$

\section{2.3 Results}

Thus, the relativistic part of the Lagrange function associated with the body $m_{n}$ can be represented in the form:

$$
\Delta L_{n}=\Delta L_{n}^{(0)}\left(\omega_{k}^{0}\right)+\Delta L_{n}^{(1)}\left(\omega_{k}^{1}\right)+\Delta L_{n}^{(2)}\left(\omega_{k}^{2}\right),
$$

where $\Delta L_{n}^{(0)}\left(\omega_{k}^{0}\right)$ independent from the components of the angular velocity $\omega_{k}$, has the form: $\Delta L_{n}^{(0)}\left(\omega_{k}^{0}\right)=\frac{1}{8 c^{2}} \dot{\bar{R}}_{n}^{4} m_{n}+$ 


$$
\begin{aligned}
& +\sum_{j \neq n} \frac{G m_{j}}{c^{2}}\left\langle\frac{3}{2} \frac{\dot{\bar{R}}_{n}^{2}}{\Delta_{n j}} m_{n}+\frac{3 \dot{\bar{R}}_{n}^{2}}{4 \Delta_{n j}^{3}}\left\{A_{n}+B_{n}+C_{n}-\frac{3}{\Delta_{n j}^{2}}\left[A_{n}\left(x_{n}-x_{j}\right)^{2}+B_{n}\left(y_{n}-y_{j}\right)^{2}+C_{n}\left(z_{n}-z_{j}\right)^{2}\right]\right\}+\right. \\
& +\frac{3}{2} \dot{\bar{R}}_{j}^{2} \frac{m_{n}}{\Delta_{n j}}+\frac{3 \dot{\bar{R}}_{j}^{2}}{4 \Delta_{n j}^{3}}\left\{A_{n}+B_{n}+C_{n}-\frac{3}{\Delta_{n j}^{2}}\left[A_{n}\left(x_{n}-x_{j}\right)^{2}+B_{n}\left(y_{n}-y_{j}\right)^{2}+C_{n}\left(z_{n}-z_{j}\right)^{2}\right]\right\}- \\
& -\frac{7}{2} \frac{\dot{\bar{R}}_{n} \cdot \dot{\bar{R}}_{j}}{\Delta_{n j}} m_{n}-\frac{7}{4} \frac{\dot{\bar{R}}_{n} \cdot \dot{\bar{R}}_{j}}{\Delta_{n j}^{3}}\left\{A_{n}+B_{n}+C_{n}-\frac{3}{\Delta_{n j}^{2}}\left[A_{n}\left(x_{n}-x_{j}\right)^{2}+B_{n}\left(y_{n}-y_{j}\right)^{2}+C_{n}\left(z_{n}-z_{j}\right)^{2}\right]\right\}- \\
& -\frac{1}{2} \dot{\bar{R}}_{n} \cdot\left(\bar{R}_{n}-\bar{R}_{j}\right) \dot{\bar{R}}_{j} \cdot\left(\bar{R}_{n}-\bar{R}_{j}\right) \frac{1}{\Delta_{n j}^{3}} m_{n}+ \\
& +\dot{\bar{R}}_{n} \cdot\left(\bar{R}_{n}-\bar{R}_{j}\right) \dot{\bar{R}}_{j} \cdot\left(\bar{R}_{n}-\bar{R}_{j}\right) \frac{15}{4 \Delta_{n j}^{7}}\left[A_{n}\left(x_{n}-x_{j}\right)^{2}+B_{n}\left(y_{n}-y_{j}\right)^{2}+C_{n}\left(z_{n}-z_{j}\right)^{2}\right]- \\
& -\frac{3 \dot{\bar{R}}_{n} \cdot\left(\bar{R}_{n}-\bar{R}_{j}\right)}{2 \Delta_{n j}^{5}}\left[\dot{x}_{j}\left(x_{n}-x_{j}\right) A_{n}+\dot{y}_{j}\left(y_{n}-y_{j}\right) B_{n}+\dot{z}_{j}\left(z_{n}-z_{j}\right) C_{n}\right]- \\
& -\frac{3 \dot{\bar{R}}_{j} \cdot\left(\bar{R}_{n}-\bar{R}_{j}\right)}{2 \Delta_{n j}^{5}}\left[\dot{x}_{n}\left(x_{n}-x_{j}\right) A_{n}+\dot{y}_{n}\left(y_{n}-y_{j}\right) B_{n}+\dot{z}_{n}\left(z_{n}-z_{j}\right) C_{n}\right]- \\
& \left.-\frac{\dot{\bar{R}}_{n} \cdot \dot{\bar{R}}_{j}}{4 \Delta_{n j}^{3}}\left(A_{n}+B_{n}+C_{n}\right)+\frac{1}{2 \Delta_{n j}^{3}}\left[\dot{x}_{n} \dot{x}_{j} A_{n}+\dot{y}_{n} \dot{y}_{j} B_{n}+\dot{z}_{n} \dot{z}_{j} C_{n}\right]\right\rangle- \\
& -\frac{1}{2 c^{2}} \sum_{j \neq n} \sum_{k \neq n} \frac{G m_{j}}{\Delta_{n j}} \frac{G m_{k}}{\Delta_{n k}}\left\{m_{n}+\frac{1}{2}\left[\frac{\left(\bar{R}_{n}-\bar{R}_{j}\right) \cdot\left(\bar{R}_{n}-\bar{R}_{k}\right)}{\Delta_{n j}^{2} \Delta_{n k}^{2}}+\frac{1}{2}\left(\frac{1}{\Delta_{n j}^{2}}+\frac{1}{\Delta_{n k}^{2}}\right)\right]\left(A_{n}+B_{n}+C_{n}\right)-\right. \\
& -\frac{1}{\Delta_{n j}^{2} \Delta_{n k}^{2}}\left[A_{n}\left(x_{n}-x_{j}\right)\left(x_{n}-x_{k}\right)+B_{n}\left(y_{n}-y_{j}\right)\left(y_{n}-y_{k}\right)+C_{n}\left(z_{n}-z_{j}\right)\left(z_{n}-z_{k}\right)\right]- \\
& -\frac{3}{2 \Delta_{n j}^{4}}\left[A_{n}\left(x_{n}-x_{j}\right)^{2}+B_{n}\left(y_{n}-y_{j}\right)^{2}+C_{n}\left(z_{n}-z_{j}\right)^{2}\right]- \\
& \left.-\frac{3}{2 \Delta_{n k}^{4}}\left[A_{n}\left(x_{n}-x_{k}\right)^{2}+B_{n}\left(y_{n}-y_{k}\right)^{2}+C_{n}\left(z_{n}-z_{k}\right)^{2}\right]\right\}- \\
& -\sum_{j \neq n} \frac{G m_{j}}{c^{2}} \frac{G m_{n}}{2 \Delta_{n j}^{2}}\left\langle m_{n}+\frac{1}{\Delta_{n j}^{2}}\left\{A_{n}+B_{n}+C_{n}-\frac{3}{\Delta_{n j}^{2}}\left[A_{n}\left(x_{n}-x_{j}\right)^{2}+B_{n}\left(y_{n}-y_{j}\right)^{2}+C_{n}\left(z_{n}-z_{j}\right)^{2}\right]\right\}\right\rangle \text {. }
\end{aligned}
$$

After the reduction of similar terms the relativistic part of the Lagrange function, which relate to the force function of gravitational interaction the body $m_{n}$ with other bodies has form:

$$
\begin{aligned}
& \Delta L_{n}^{(0)}\left(\omega_{k}^{0}\right)=\frac{1}{8 c^{2}} \dot{\bar{R}}_{n}^{4} m_{n}+\frac{1}{c^{2}} \sum_{j \neq n} G m_{j}\left\langle\left(\frac{3}{2} \frac{\dot{\bar{R}}_{n}^{2}}{\Delta_{n j}}+\frac{3}{2} \frac{\dot{\bar{R}}_{j}^{2}}{\Delta_{n j}}-\frac{7}{2} \frac{\dot{\bar{R}}_{n} \cdot \dot{\bar{R}}_{j}}{\Delta_{n j}}\right)\right. \\
& \left\langle m_{n}+\frac{1}{2 \Delta_{n j}^{2}}\left\{A_{n}+B_{n}+C_{n}-\frac{3}{\Delta_{n j}^{2}}\left[A_{n}\left(x_{n}-x_{j}\right)^{2}+B_{n}\left(y_{n}-y_{j}\right)^{2}+C_{n}\left(z_{n}-z_{j}\right)^{2}\right]\right\}\right\rangle-
\end{aligned}
$$




$$
\begin{aligned}
& -\frac{\dot{\bar{R}}_{n} \cdot\left(\bar{R}_{n}-\bar{R}_{j}\right) \dot{\bar{R}}_{j} \cdot\left(\bar{R}_{n}-\bar{R}_{j}\right)}{2 \Delta_{n j}^{3}}\left\{m_{n}-\frac{15}{2 \Delta_{n j}^{4}}\left[A_{n}\left(x_{n}-x_{j}\right)^{2}+B_{n}\left(y_{n}-y_{j}\right)^{2}+C_{n}\left(z_{n}-z_{j}\right)^{2}\right]\right\}- \\
& -\frac{3 \dot{\bar{R}}_{n} \cdot\left(\bar{R}_{n}-\bar{R}_{j}\right)}{2 \Delta_{n j}^{5}}\left[\dot{x}_{j}\left(x_{n}-x_{j}\right) A_{n}+\dot{y}_{j}\left(y_{n}-y_{j}\right) B_{n}+\dot{z}_{j}\left(z_{n}-z_{j}\right) C_{n}\right]- \\
& -\frac{3 \dot{\bar{R}}_{j} \cdot\left(\bar{R}_{n}-\bar{R}_{j}\right)}{2 \Delta_{n j}^{5}}\left[\dot{x}_{n}\left(x_{n}-x_{j}\right) A_{n}+\dot{y}_{n}\left(y_{n}-y_{j}\right) B_{n}+\dot{z}_{n}\left(z_{n}-z_{j}\right) C_{n}\right]- \\
& \left.-\frac{\dot{\bar{R}}_{n} \cdot \dot{\bar{R}}_{j}}{4 \Delta_{n j}^{3}}\left(A_{n}+B_{n}+C_{n}\right)+\frac{1}{2 \Delta_{n j}^{3}}\left[\dot{x}_{n} \dot{x}_{j} A_{n}+\dot{y}_{n} \dot{y}_{j} B_{n}+\dot{z}_{n} \dot{z}_{j} C_{n}\right]\right\rangle- \\
& -\frac{1}{2 c^{2}} \sum_{j \neq n} \sum_{k \neq n} \frac{G m_{j}}{\Delta_{n j}} \frac{G m_{k}}{\Delta_{n k}}\left\{m_{n}+\frac{1}{2}\left[\frac{\left(\bar{R}_{n}-\bar{R}_{j}\right) \cdot\left(\bar{R}_{n}-\bar{R}_{k}\right)}{\Delta_{n j}^{2} \Delta_{n k}^{2}}+\frac{1}{2}\left(\frac{1}{\Delta_{n j}^{2}}+\frac{1}{\Delta_{n k}^{2}}\right)\right]\left(A_{n}+B_{n}+C_{n}\right)-\right. \\
& -\frac{1}{\Delta_{n j}^{2} \Delta_{n k}^{2}}\left[A_{n}\left(x_{n}-x_{j}\right)\left(x_{n}-x_{k}\right)+B_{n}\left(y_{n}-y_{j}\right)\left(y_{n}-y_{k}\right)+C_{n}\left(z_{n}-z_{j}\right)\left(z_{n}-z_{k}\right)\right]- \\
& -\frac{3}{2 \Delta_{n j}^{4}}\left[A_{n}\left(x_{n}-x_{j}\right)^{2}+B_{n}\left(y_{n}-y_{j}\right)^{2}+C_{n}\left(z_{n}-z_{j}\right)^{2}\right]- \\
& \left.-\frac{3}{2 \Delta_{n k}^{4}}\left[A_{n}\left(x_{n}-x_{k}\right)^{2}+B_{n}\left(y_{n}-y_{k}\right)^{2}+C_{n}\left(z_{n}-z_{k}\right)^{2}\right]\right\}-\frac{G m_{n}}{2 c^{2}} \sum_{j \neq n} \frac{G m_{j}}{\Delta_{n j}}\left\langle\frac{m_{n}}{\Delta_{n j}}+\right. \\
& \left.+\frac{1}{\Delta_{n j}^{3}}\left\{A_{n}+B_{n}+C_{n}-\frac{3}{\Delta_{n j}^{2}}\left[A_{n}\left(x_{n}-x_{j}\right)^{2}+B_{n}\left(y_{n}-y_{j}\right)^{2}+C_{n}\left(z_{n}-z_{j}\right)^{2}\right]\right\}\right) \text {. }
\end{aligned}
$$

If the body $m_{n}$ is spherically symmetric, that is $A_{n}=B_{n}=C_{n}=I_{n}$, then $\Delta L_{n}^{(0)}\left(\omega_{k}^{0}\right)$ becomes:

$$
\begin{aligned}
& \Delta L_{n}^{(0)}\left(\omega_{k}^{0}\right)=\frac{1}{8 c^{2}} \dot{\bar{R}}_{n}^{4} m_{n}+\sum_{j \neq n} \frac{G m_{j}}{c^{2}}\left[\frac{3}{2} \frac{\dot{\bar{R}}_{n}^{2}}{\Delta_{n j}} m_{n}-\frac{7}{2} \frac{\dot{\bar{R}}_{n} \cdot \dot{\bar{R}}_{j}}{\Delta_{n j}} m_{n}-\right. \\
& \left.-\frac{1}{2} \dot{\bar{R}}_{n} \cdot\left(\bar{R}_{n}-\bar{R}_{j}\right) \dot{\bar{R}}_{j} \cdot\left(\bar{R}_{n}-\bar{R}_{j}\right) \frac{1}{\Delta_{n j}^{3}} m_{n}+\dot{\bar{R}}_{n} \cdot\left(\bar{R}_{n}-\bar{R}_{j}\right) \dot{\bar{R}}_{j} \cdot\left(\bar{R}_{n}-\bar{R}_{j}\right) \frac{15}{4 \Delta_{n j}^{5}} I_{n}-\frac{3 \dot{\bar{R}}_{n} \cdot \dot{\bar{R}}_{j}}{4 \Delta_{n j}^{3}} I_{n}\right] .
\end{aligned}
$$

An additional part $\Delta L_{n}^{(1)}\left(\omega_{k}^{1}\right)$ that depends linearly on the components $\omega_{k}$ has the form:

$$
\begin{aligned}
& \Delta L_{n}^{(1)}\left(\omega_{k}^{1}\right)=\sum_{j \neq n} \frac{G m_{j}}{c^{2}}\left\langle-\frac{3}{2 \Delta_{n j}^{3}}\left\{\bar{H}_{n} \cdot\left(\bar{R}_{n}-\bar{R}_{j}\right) \times \dot{\bar{R}}_{n}+\right.\right. \\
& +\left(C_{n}-B_{n}\right) \omega_{1}\left[\left(y_{n}-y_{j}\right) \dot{z}_{n}+\left(z_{n}-z_{j}\right) \dot{y}_{n}\right]+\left(A_{n}-C_{n}\right) \omega_{2}\left[\left(z_{n}-z_{j}\right) \dot{x}_{n}+\left(x_{n}-x_{j}\right) \dot{z}_{n}\right]+ \\
& \left.+\left(B_{n}-A_{n}\right) \omega_{3}\left[\left(x_{n}-x_{j}\right) \dot{y}_{n}+\left(y_{n}-y_{j}\right) \dot{x}_{n}\right]\right\}+ \\
& +\frac{7}{4 \Delta_{n j}^{3}}\left\{\bar{H}_{n} \cdot\left(\bar{R}_{n}-\bar{R}_{j}\right) \times \dot{\bar{R}}_{j}+\left(C_{n}-B_{n}\right) \omega_{1}\left[\left(y_{n}-y_{j}\right) \dot{z}_{j}+\left(z_{n}-z_{j}\right) \dot{y}_{j}\right]+\right.
\end{aligned}
$$




$$
\begin{aligned}
& \left.+\left(A_{n}-C_{n}\right) \omega_{2}\left[\left(z_{n}-z_{j}\right) \dot{x}_{j}+\left(x_{n}-x_{j}\right) \dot{z}_{j}\right]+\left(B_{n}-A_{n}\right) \omega_{3}\left[\left(x_{n}-x_{j}\right) \dot{y}_{j}+\left(y_{n}-y_{j}\right) \dot{x}_{j}\right]\right\}+ \\
& +\frac{3 \dot{\bar{R}}_{j} \cdot\left(\bar{R}_{n}-\bar{R}_{j}\right)}{2 \Delta_{n j}^{5}}\left[\left(x_{n}-x_{j}\right)\left(y_{n}-y_{j}\right) \omega_{3}\left(B_{n}-A_{n}\right)+\left(z_{n}-z_{j}\right)\left(x_{n}-x_{j}\right) \omega_{2}\left(A_{n}-C_{n}\right)+\right. \\
& \left.+\left(y_{n}-y_{j}\right)\left(z_{n}-z_{j}\right) \omega_{1}\left(C_{n}-B_{n}\right)\right]+ \\
& +\frac{1}{4 \Delta_{n j}^{3}}\left\{\bar{H}_{n} \cdot\left(\bar{R}_{n}-\bar{R}_{j}\right) \times \dot{\bar{R}}_{j}-\right. \\
& -\left(C_{n}-B_{n}\right) \omega_{1}\left[\dot{y}_{j}\left(z_{n}-z_{j}\right)+\dot{z}_{j}\left(y_{n}-y_{j}\right)\right]-\left(A_{n}-C_{n}\right) \omega_{2}\left[\dot{z}_{j}\left(x_{n}-x_{j}\right)+\dot{x}_{j}\left(z_{n}-z_{j}\right)\right]- \\
& \left.\left.-\left(B_{n}-A_{n}\right) \omega_{3}\left[\dot{x}_{j}\left(y_{n}-y_{j}\right)+\dot{y}_{j}\left(x_{n}-x_{j}\right)\right]\right\}\right\rangle .
\end{aligned}
$$

After the reduction of similar terms an additional part $\Delta L_{n}^{(1)}\left(\omega_{k}^{1}\right)$ has the form:

$$
\begin{aligned}
& \Delta L_{n}^{(1)}\left(\omega_{k}^{1}\right)=-\sum_{j \neq n} \frac{G m_{j}}{c^{2}} \frac{1}{\Delta_{n j}^{3}}\left\{\bar{H}_{n} \cdot\left(\bar{R}_{n}-\bar{R}_{j}\right) \times\left(\frac{3}{2} \dot{\bar{R}}_{n}-2 \dot{\bar{R}}_{j}\right)+\right. \\
& +\frac{3}{2}\left(C_{n}-B_{n}\right) \omega_{1}\left[\left(y_{n}-y_{j}\right)\left(\dot{z}_{n}-\dot{z}_{j}\right)+\left(z_{n}-z_{j}\right)\left(\dot{y}_{n}-\dot{y}_{j}\right)\right]+ \\
& +\frac{3}{2}\left(A_{n}-C_{n}\right) \omega_{2}\left[\left(z_{n}-z_{j}\right)\left(\dot{x}_{n}-\dot{x}_{j}\right)+\left(x_{n}-x_{j}\right)\left(\dot{z}_{n}-\dot{z}_{j}\right)\right]+ \\
& +\frac{3}{2}\left(B_{n}-A_{n}\right) \omega_{3}\left[\left(x_{n}-x_{j}\right)\left(\dot{y}_{n}-\dot{y}_{j}\right)+\left(y_{n}-y_{j}\right)\left(\dot{x}_{n}-\dot{x}_{j}\right)\right]- \\
& -\frac{3 \dot{\bar{R}}_{j} \cdot\left(\bar{R}_{n}-\bar{R}_{j}\right)}{2 \Delta_{n j}^{2}}\left[\left(x_{n}-x_{j}\right)\left(y_{n}-y_{j}\right) \omega_{3}\left(B_{n}-A_{n}\right)+\left(z_{n}-z_{j}\right)\left(x_{n}-x_{j}\right) \omega_{2}\left(A_{n}-C_{n}\right)+\right. \\
& \left.\left.+\left(y_{n}-y_{j}\right)\left(z_{n}-z_{j}\right) \omega_{1}\left(C_{n}-B_{n}\right)\right]\right\} .
\end{aligned}
$$

It is Lagrange function for the geodetic rotation of the rigid body, which was received in the previous investigations (Eroshkin, Pashkevich, 1997), (Pashkevich, 2000).

The first line turned out to be the relativistic components of the Lagrange function, which relate to the body $m_{n}$ as a point mass or as spherically symmetric:

$$
\Delta L_{n}^{(1)}\left(\omega_{k}^{1}\right)=-\sum_{j \neq n} \frac{G m_{j}}{c^{2}} \frac{1}{\Delta_{n j}^{3}} \bar{H}_{n} \cdot\left(\bar{R}_{n}-\bar{R}_{j}\right) \times\left(\frac{3}{2} \dot{\bar{R}}_{n}-2 \dot{\bar{R}}_{j}\right) .
$$

An additional part $\Delta L_{n}^{(2)}\left(\omega_{k}^{2}\right)$ that depends square on the components $\omega_{k}^{2}$ has the form:

$$
\begin{aligned}
& \Delta L_{n}^{(2)}\left(\omega_{k}^{2}\right)=\frac{1}{4 c^{2}} \dot{\bar{R}}_{n}^{2}\left(A_{n} \omega_{1}^{2}+B_{n} \omega_{2}^{2}+C_{n} \omega_{3}^{2}\right)+\sum_{j \neq n} \frac{G m_{j}}{c^{2}}\left[\frac{3}{2 \Delta_{n j}}\left(A_{n} \omega_{1}^{2}+B_{n} \omega_{2}^{2}+C_{n} \omega_{3}^{2}\right)+\right. \\
& +\left(\dot{x}_{n} \omega_{2}-\dot{y}_{n} \omega_{1}\right)^{2} \frac{A_{n}+B_{n}-C_{n}}{4}+\left(\dot{x}_{n} \omega_{3}-\dot{z}_{n} \omega_{1}\right)^{2} \frac{C_{n}+A_{n}-B_{n}}{4}+ \\
& \left.+\left(\dot{y}_{n} \omega_{3}-\dot{z}_{n} \omega_{2}\right)^{2} \frac{B_{n}+C_{n}-A_{n}}{4}\right]=\frac{1}{2} \bar{H}_{n} \cdot \bar{\omega} \frac{1}{c^{2}}\left(\frac{\dot{\bar{R}}_{n}^{2}}{2}+3 \sum_{j \neq n} \frac{G m_{j}}{\Delta_{n j}}\right)+ \\
& +\sum_{j \neq n} \frac{G m_{j}}{c^{2}}\left[\left(\dot{x}_{n} \omega_{2}-\dot{y}_{n} \omega_{1}\right)^{2} \frac{A_{n}+B_{n}-C_{n}}{4}+\left(\dot{x}_{n} \omega_{3}-\dot{z}_{n} \omega_{1}\right)^{2} \frac{C_{n}+A_{n}-B_{n}}{4}+\right.
\end{aligned}
$$




$$
\left.+\left(\dot{y}_{n} \omega_{3}-\dot{z}_{n} \omega_{2}\right)^{2} \frac{B_{n}+C_{n}-A_{n}}{4}\right] .
$$

If the body $m_{n}$ is spherically symmetric, then $\Delta L_{n}^{(2)}\left(\omega_{k}^{2}\right)$ becomes:

$$
\Delta L_{n}^{(2)}\left(\omega_{k}^{2}\right)=\frac{1}{c^{2}}\left[\frac{1}{2} \bar{H}_{n} \cdot \bar{\omega}\left(\frac{\dot{\bar{R}}_{n}^{2}}{2}+3 \sum_{j \neq n} \frac{G m_{j}}{\Delta_{n j}}\right)+\frac{I_{n}}{4}\left(\dot{\bar{R}}_{n} \times \bar{\omega}\right)^{2} \sum_{j \neq n} G m_{j}\right] \text {. }
$$

\section{DERIVATION OF THE DIFFERENTIAL EQUATIONS}

The differential equations of the rigid body rotation are derived in the terms of the Rodrigues - Hamilton parameters.

Lagrange differential equations of the second kind have the form:

$$
\frac{d}{d t} \frac{\partial L}{\partial \dot{\lambda}_{i}}-\frac{\partial L}{\partial \lambda_{i}}=0, \quad i=0,1,2,3
$$

where $L=T+U+\Delta L, T=\frac{1}{2} \bar{H}_{n} \cdot \bar{\omega}=\frac{1}{2}\left(A_{n} \omega_{1}^{2}+B_{n} \omega_{2}^{2}+C_{n} \omega_{3}^{2}\right), T$ is the kinetic energy of the rotational motion of the rigid body; $U$ is the force function of the gravitational interaction of the rigid body with the disturbing bodies; $\Delta L$ is the additional part of the Lagrange function, which generating geodetic perturbations (2.10). This expression in other form is:

$$
\Delta L=\bar{H}_{n}\left(\bar{\sigma}_{n}+\bar{\sigma}_{R}\right)=\bar{H}_{n} \bar{\sigma}_{g},
$$

where $\bar{\sigma}_{n}=-\sum_{j \neq n} \frac{G m_{j}}{c^{2} \Delta_{n j}^{3}}\left(\bar{R}_{n}-\bar{R}_{j}\right) \times\left(\frac{3}{2} \dot{\bar{R}}_{n}-2 \dot{\bar{R}}_{j}\right)$;

$$
\begin{aligned}
\bar{H}_{n} \bar{\sigma}_{R}=-\frac{3}{2} \sum_{j \neq n} \frac{G m_{j}}{c^{2} \Delta_{n j}^{3}}\{ & \left(C_{n}-B_{n}\right) \omega_{1}\left[\left(y_{n}-y_{j}\right)\left(\dot{z}_{n}-\dot{z}_{j}\right)+\left(z_{n}-z_{j}\right)\left(\dot{y}_{n}-\dot{y}_{j}\right)\right]+ \\
& +\left(A_{n}-C_{n}\right) \omega_{2}\left[\left(z_{n}-z_{j}\right)\left(\dot{x}_{n}-\dot{x}_{j}\right)+\left(x_{n}-x_{j}\right)\left(\dot{z}_{n}-\dot{z}_{j}\right)\right]+ \\
& +\left(B_{n}-A_{n}\right) \omega_{3}\left[\left(x_{n}-x_{j}\right)\left(\dot{y}_{n}-\dot{y}_{j}\right)+\left(y_{n}-y_{j}\right)\left(\dot{x}_{n}-\dot{x}_{j}\right)\right]- \\
& -\frac{\dot{\bar{R}}_{j} \cdot\left(\bar{R}_{n}-\bar{R}_{j}\right)}{\Delta_{n j}^{2}}\left[\left(C_{n}-B_{n}\right) \omega_{1}\left(y_{n}-y_{j}\right)\left(z_{n}-z_{j}\right)+\right. \\
& \left.\left.+\left(A_{n}-C_{n}\right) \omega_{2}\left(z_{n}-z_{j}\right)\left(x_{n}-x_{j}\right)+\left(B_{n}-A_{n}\right) \omega_{3}\left(x_{n}-x_{j}\right)\left(y_{n}-y_{j}\right)\right]\right\} .
\end{aligned}
$$

Thus relations for relativistic angular velocity vectors have forms:

$$
\begin{aligned}
\bar{\sigma}_{n}=-\frac{1}{c^{2}} \sum_{j \neq n} \frac{G m_{j}}{\Delta_{n j}^{3}}\left\{\left[y_{n j}\left(\frac{3}{2} \dot{z}_{n}-2 \dot{z}_{j}\right)-z_{n j}\left(\frac{3}{2} \dot{y}_{n}-2 \dot{y}_{j}\right)\right] \bar{i}_{1}+\right. \\
+\left[z_{n j}\left(\frac{3}{2} \dot{x}_{n}-2 \dot{x}_{j}\right)-x_{n j}\left(\frac{3}{2} \dot{z}_{n}-2 \dot{z}_{j}\right)\right] \bar{i}_{2}+ \\
\left.+\left[x_{n j}\left(\frac{3}{2} \dot{y}_{n}-2 \dot{y}_{j}\right)-y_{n j}\left(\frac{3}{2} \dot{x}_{n}-2 \dot{x}_{j}\right)\right] \dot{i}_{3}\right\} ;
\end{aligned}
$$


$\bar{\sigma}_{R}=-\frac{3}{2} \sum_{j \neq n} \frac{G m_{j}}{c^{2} \Delta_{n j}^{3}}\left\{\alpha_{n}\left[y_{n j} \dot{z}_{n j}+z_{n j} \dot{y}_{n j}-\frac{\dot{\bar{R}}_{j} \cdot \bar{\Delta}_{n j}}{\Delta_{n j}^{2}} y_{n j} z_{n j}\right] \overline{\dot{i}}_{1}-\right.$

$\left.-\beta_{n}\left[z_{n j} \dot{x}_{n j}+x_{n j} \dot{z}_{n j}-\frac{\dot{\bar{R}}_{j} \cdot \bar{\Delta}_{n j}}{\Delta_{n j}^{2}} z_{n j} x_{n j}\right] \overline{\dot{z}}_{2}+\gamma_{n}\left[x_{n j} \dot{y}_{n j}+y_{n j} \dot{x}_{n j}-\frac{\dot{\bar{R}}_{j} \cdot \bar{\Delta}_{n j}}{\Delta_{n j}^{2}} x_{n j} y_{n j}\right] \overline{\dot{i}_{3}}\right\}$.

Here $\alpha_{n}=\left(C_{n}-B_{n}\right) / A_{n}, \beta_{n}=\left(C_{n}-A_{n}\right) / B_{n}, \gamma_{n}=\left(B_{n}-A_{n}\right) / C_{n} ; x_{n j}=x_{n}-x_{j}, y_{n j}=y_{n}-y_{j}$, $z_{n j}=z_{n}-z_{j}, \dot{x}_{n j}=\dot{x}_{n}-\dot{x}_{j}, \dot{y}_{n j}=\dot{y}_{n}-\dot{y}_{j}, \dot{z}_{n j}=\dot{z}_{n}-\dot{z}_{j} ; \bar{\Delta}_{n j}=\bar{R}_{n}-\bar{R}_{j} ; \Delta_{n j}=\left|\bar{R}_{n}-\bar{R}_{j}\right|$.

The first derivatives of the relativistic angular velocity vectors are given by the following expressions:

$$
\begin{aligned}
& \dot{\bar{\sigma}}_{n}=-\frac{1}{c^{2}} \sum_{j \neq n} \frac{G m_{j}}{\Delta_{n j}^{3}}\left\{\left[-3 \frac{\dot{\Delta}_{n j}}{\Delta_{n j}} \bar{\Delta}_{n j} \times\left(\frac{3}{2} \dot{\bar{R}}_{n}-2 \dot{\bar{R}}_{j}\right)+\dot{\bar{\Delta}}_{n j} \times\left(\frac{3}{2} \dot{\bar{R}}_{n}-2 \dot{\bar{R}}_{j}\right)+\right.\right.\left.\left.+\bar{\Delta}_{n j} \times\left(\frac{3}{2} \ddot{\bar{R}}_{n}-2 \ddot{\bar{R}}_{j}\right)\right]\right\} ; \\
& \dot{\bar{\sigma}}_{R}=-\frac{3}{2 c^{2}} \sum_{j \neq n} \frac{G m_{j}}{\Delta_{n j}^{3}}\left\{\alpha _ { n } \left[-3 \frac{\dot{\Delta}_{n j}}{\Delta_{n j}}\left(y_{n j} \dot{z}_{n j}+z_{n j} \dot{y}_{n j}\right)+2 \dot{y}_{n j} \dot{z}_{n j}+y_{n j} \ddot{z}_{n j}+z_{n j} \ddot{y}_{n j}-\right.\right. \\
&\left.-\frac{1}{\Delta_{n j}^{2}}\left(\dot{\bar{R}}_{j} \cdot \bar{\Delta}_{n j}\left(-5 \frac{\dot{\Delta}_{n j}}{\Delta_{n j}} y_{n j} z_{n j}+\dot{y}_{n j} z_{n j}+y_{n j} \dot{z}_{n j}\right)+\left(\dot{\bar{R}}_{j} \cdot \dot{\bar{\Delta}}_{n j}+\ddot{\bar{R}}_{j} \cdot \bar{\Delta}_{n j}\right) y_{n j} z_{n j}\right)\right] \dot{\bar{q}}_{1}- \\
&-\beta_{n}\left[-3 \frac{\dot{\Delta}_{n j}}{\Delta_{n j}}\left(z_{n j} \dot{x}_{n j}+x_{n j} \dot{z}_{n j}\right)+2 \dot{z}_{n j} \dot{x}_{n j}+z_{n j} \ddot{x}_{n j}+x_{n j} \ddot{z}_{n j}-\right. \\
&\left.-\frac{1}{\Delta_{n j}^{2}}\left(\dot{\bar{R}}_{j} \cdot \bar{\Delta}_{n j}\left(-5 \frac{\dot{\Delta}_{n j}}{\Delta_{n j}} z_{n j} x_{n j}+\dot{z}_{n j} x_{n j}+z_{n j} \dot{x}_{n j}\right)+\left(\dot{\bar{R}}_{j} \cdot \dot{\bar{\Delta}}_{n j}+\ddot{\bar{R}}_{j} \cdot \bar{\Delta}_{n j}\right) z_{n j} x_{n j}\right)\right] \overline{\dot{z}}_{2}+ \\
&\left.\left.+\frac{1}{\Delta_{n j}^{2}}\left(\dot{\bar{R}}_{j} \cdot \bar{\Delta}_{n j}\left(-5 \frac{\dot{\Delta}_{n j}}{\Delta_{n j}} x_{n j} y_{n j}+\dot{x}_{n j} y_{n j}+x_{n j} \dot{y}_{n j}\right)+\left(\dot{\bar{R}}_{j} \cdot \dot{\bar{\Delta}}_{n j}+\ddot{\bar{R}}_{j} \cdot \bar{\Delta}_{n j}\right) x_{n j} y_{n j}\right)\right] \dot{\vec{j}}_{3}\right\} .
\end{aligned}
$$

To define new operators:

$$
\begin{aligned}
& \langle\rangle_{0}=\lambda_{0}\{\}_{0}+\lambda_{1}\{\}_{1}+\lambda_{2}\{\}_{2}+\lambda_{3}\{\}_{3}, \\
& \langle\rangle_{1}=\lambda_{1}\{\}_{0}-\lambda_{0}\{\}_{1}-\lambda_{3}\{\}_{2}+\lambda_{2}\{\}_{3}, \\
& \langle\rangle_{2}=\lambda_{2}\{\}_{0}+\lambda_{3}\{\}_{1}-\lambda_{0}\{\}_{2}-\lambda_{1}\{\}_{3}, \\
& \langle\rangle_{3}=\lambda_{3}\{\}_{0}-\lambda_{2}\{\}_{1}+\lambda_{1}\{\}_{2}-\lambda_{0}\{\}_{3} ;
\end{aligned}
$$

where \{\}$_{i}=\frac{d}{d t} \frac{\partial}{\partial \dot{\lambda}_{i}}-\frac{\partial}{\partial \lambda_{i}}$; 


$$
\begin{aligned}
& ()_{0}=\lambda_{0} \frac{\partial}{\partial \dot{\lambda}_{0}}+\lambda_{1} \frac{\partial}{\partial \dot{\lambda}_{1}}+\lambda_{2} \frac{\partial}{\partial \dot{\lambda}_{2}}+\lambda_{3} \frac{\partial}{\partial \dot{\lambda}_{3}}, \\
& ()_{1}=\lambda_{1} \frac{\partial}{\partial \dot{\lambda}_{0}}-\lambda_{0} \frac{\partial}{\partial \dot{\lambda}_{1}}-\lambda_{3} \frac{\partial}{\partial \dot{\lambda}_{2}}+\lambda_{2} \frac{\partial}{\partial \dot{\lambda}_{3}}, \\
& ()_{2}=\lambda_{2} \frac{\partial}{\partial \dot{\lambda}_{0}}+\lambda_{3} \frac{\partial}{\partial \dot{\lambda}_{1}}-\lambda_{0} \frac{\partial}{\partial \dot{\lambda}_{2}}-\lambda_{1} \frac{\partial}{\partial \dot{\lambda}_{3}}, \\
& ()_{3}=\lambda_{3} \frac{\partial}{\partial \dot{\lambda}_{0}}-\lambda_{2} \frac{\partial}{\partial \dot{\lambda}_{1}}+\lambda_{1} \frac{\partial}{\partial \dot{\lambda}_{2}}-\lambda_{0} \frac{\partial}{\partial \dot{\lambda}_{3}} \\
& {[]_{0}=\lambda_{0} \frac{\partial}{\partial \lambda_{0}}+\lambda_{1} \frac{\partial}{\partial \lambda_{1}}+\lambda_{2} \frac{\partial}{\partial \lambda_{2}}+\lambda_{3} \frac{\partial}{\partial \lambda_{3}},} \\
& {[]_{1}=\lambda_{1} \frac{\partial}{\partial \lambda_{0}}-\lambda_{0} \frac{\partial}{\partial \lambda_{1}}-\lambda_{3} \frac{\partial}{\partial \lambda_{2}}+\lambda_{2} \frac{\partial}{\partial \lambda_{3}},} \\
& {[]_{2}=\lambda_{2} \frac{\partial}{\partial \lambda_{0}}+\lambda_{3} \frac{\partial}{\partial \lambda_{1}}-\lambda_{0} \frac{\partial}{\partial \lambda_{2}}-\lambda_{1} \frac{\partial}{\partial \lambda_{3}},} \\
& {[]_{3}=\lambda_{3} \frac{\partial}{\partial \lambda_{0}}-\lambda_{2} \frac{\partial}{\partial \lambda_{1}}+\lambda_{1} \frac{\partial}{\partial \lambda_{2}}-\lambda_{0} \frac{\partial}{\partial \lambda_{3}} .}
\end{aligned}
$$

The force function (2.6) is independent from $\dot{\bar{R}}_{n}^{*}$, accordingly one is independent from $\dot{\lambda}_{i}$ :

$$
\frac{\partial U}{\partial \dot{\lambda}_{i}}=0 \text {. }
$$

From (3.1) is received: $\{T\}_{i}=\frac{d}{d t} \frac{\partial T}{\partial \dot{\lambda}_{i}}-\frac{\partial T}{\partial \lambda_{i}}=-\frac{d}{d t} \frac{\partial \Delta L}{\partial \dot{\lambda}_{i}}+\frac{\partial(U+\Delta L)}{\partial \lambda_{i}}$, then

$$
\langle T\rangle_{i}=-\langle\Delta L\rangle_{i}+[U]_{i} .
$$

After apply (see Appendix B: (16-19)) operator (3.2) to the kinetic energy has a form:

$$
\begin{aligned}
& \langle T\rangle_{0}=-4\left(A_{n} \omega_{1}^{2}+B_{n} \omega_{2}^{2}+C_{n} \omega_{3}^{2}\right)=-8 T, \\
& \langle T\rangle_{1}=2\left(-A_{n} \dot{\omega}_{1}+B_{n} \omega_{2} \omega_{3}-C_{n} \omega_{2} \omega_{3}\right), \\
& \langle T\rangle_{2}=2\left(-B_{n} \dot{\omega}_{2}+C_{n} \omega_{1} \omega_{3}-A_{n} \omega_{1} \omega_{3}\right), \\
& \langle T\rangle_{3}=2\left(-C_{n} \dot{\omega}_{3}+A_{n} \omega_{1} \omega_{2}-B_{n} \omega_{1} \omega_{2}\right) .
\end{aligned}
$$

From (3.5) and (3.6) received the first derivatives of the angular velocity components are given by the following equations:

$$
\begin{aligned}
& \dot{\omega}_{1}=-\alpha_{n} \omega_{2} \omega_{3}+\frac{1}{2 A_{n}}\left(\langle\Delta L\rangle_{1}-[\mathrm{U}]_{1}\right), \\
& \dot{\omega}_{2}=\beta_{n} \omega_{1} \omega_{3}+\frac{1}{2 B_{n}}\left(\langle\Delta L\rangle_{2}-[\mathrm{U}]_{2}\right), \\
& \dot{\omega}_{3}=-\gamma_{n} \omega_{1} \omega_{2}+\frac{1}{2 C_{n}}\left(\langle\Delta L\rangle_{3}-[\mathrm{U}]_{3}\right), \\
& \text { where }\langle\Delta L\rangle_{i}=\langle\bar{H}\rangle_{i} \bar{\sigma}_{g}+(\bar{H})_{i} \dot{\bar{\sigma}}_{g}-\bar{H}\left[\bar{\sigma}_{g}\right]_{i} ;
\end{aligned}
$$

After apply (see Appendix B: (B.5)) operator (3.2) to the angular momentum vector of the rotational motion of the rigid body has a form: 


$$
\begin{aligned}
& \langle\bar{H}\rangle_{0}=-2 \bar{H},\langle\bar{H}\rangle_{1}=2\left(B_{n} \omega_{3} \vec{i}_{2}-C_{n} \omega_{2} \vec{i}_{3}\right), \\
& \langle\bar{H}\rangle_{2}=2\left(-A_{n} \omega_{3} \vec{i}_{1}+C_{n} \omega_{1} \vec{i}_{3}\right),\langle\bar{H}\rangle_{3}=2\left(A_{n} \omega_{2} \vec{i}_{1}+B_{n} \omega_{1} \vec{i}_{2}\right) ;
\end{aligned}
$$

After apply (see Appendix B) operator (3.3) to the angular momentum vector of the rotational motion of the rigid body has a form:

$$
(\bar{H})_{0}=0,(\bar{H})_{1}=-2 A_{n} \vec{i}_{1},(\bar{H})_{2}=-2 B_{n} \vec{i}_{2},(\bar{H})_{3}=-2 C_{n} \vec{i}_{3} .
$$

After apply operators (3.2), (3.3) and (3.4) to $\Delta \mathrm{L}$ the first derivatives of the angular velocity components are given by the following equations:

$$
\begin{aligned}
& \dot{\omega}_{1}+\dot{\sigma}_{1 g}=-\alpha_{n}\left\{\omega_{2} \omega_{3}+\omega_{2} \sigma_{3 n}+\sigma_{2 n} \omega_{3}-\omega_{2} \frac{\sigma_{3 R}}{\gamma_{n}}-\frac{\sigma_{2 R}}{\beta_{n}} \omega_{3}+\omega_{1} F_{1}\right\}-\frac{1}{2 A_{n}}[\mathrm{U}]_{1}, \\
& \dot{\omega}_{2}+\dot{\sigma}_{2 g}=\beta_{n}\left\{\omega_{1} \omega_{3}+\omega_{1} \sigma_{3 n}+\sigma_{1 n} \omega_{3}+\omega_{1} \frac{\sigma_{3 R}}{\gamma_{n}}-\frac{\sigma_{1 R}}{\alpha_{n}} \omega_{3}+\omega_{2} F_{2}\right\}-\frac{1}{2 B_{n}}[\mathrm{U}]_{2}, \\
& \dot{\omega}_{3}+\dot{\sigma}_{3 g}=-\gamma_{n}\left\{\omega_{1} \omega_{2}+\omega_{1} \sigma_{2 n}+\sigma_{1 n} \omega_{2}+\omega_{1} \frac{\sigma_{2 R}}{\beta_{n}}+\frac{\sigma_{1 R}}{\alpha_{n}} \omega_{2}+\omega_{3} F_{3}\right\}-\frac{1}{2 C_{n}}[\mathrm{U}]_{3} ;
\end{aligned}
$$

where $\bar{\sigma}_{g}=\bar{\sigma}_{n}+\bar{\sigma}_{R}, \dot{\bar{\sigma}}_{g}=\dot{\bar{\sigma}}_{n}+\dot{\bar{\sigma}}_{R}$,

$$
\begin{aligned}
& F_{1}=\frac{3}{2} \sum_{j \neq n} \frac{G m_{j}}{c^{2} \Delta_{n j}^{3}}\left[\frac{\dot{\bar{R}}_{j} \cdot \bar{\Delta}_{n j}}{\Delta_{n j}^{2}}\left(y_{n j}^{2}-z_{n j}^{2}\right)-2\left(y_{n j} \dot{y}_{n j}-z_{n j} \dot{z}_{n j}\right)\right], \\
& F_{2}=\frac{3}{2} \sum_{j \neq n} \frac{G m_{j}}{c^{2} \Delta_{n j}^{3}}\left[\frac{\dot{\bar{R}}_{j} \cdot \bar{\Delta}_{n j}}{\Delta_{n j}^{2}}\left(z_{n j}^{2}-x_{n j}^{2}\right)-2\left(z_{n j} \dot{z}_{n j}-x_{n j} \dot{x}_{n j}\right)\right], \\
& F_{3}=\frac{3}{2} \sum_{j \neq n} \frac{G m_{j}}{c^{2} \Delta_{n j}^{3}}\left[\frac{\dot{\bar{R}}_{j}}{\Delta_{n j}^{2}} \bar{\Delta}_{n j}\left(x_{n j}^{2}-y_{n j}^{2}\right)-2\left(x_{n j} \dot{x}_{n j}-y_{n j} \dot{y}_{n j}\right)\right] .
\end{aligned}
$$

The differential equations, determining the rigid body rotation in the terms of the Rodrigues Hamilton parameters are obtained from expressions of the angular velocity vector of the rigid body:

$$
\left(\begin{array}{c}
0 \\
\omega_{1} \\
\omega_{2} \\
\omega_{3}
\end{array}\right)=2 K\left(\begin{array}{c}
\dot{\lambda}_{0} \\
\dot{\lambda}_{1} \\
\dot{\lambda}_{2} \\
\dot{\lambda}_{3}
\end{array}\right), K=\left(\begin{array}{cccc}
-\lambda_{0} & -\lambda_{1} & -\lambda_{2} & -\lambda_{3} \\
-\lambda_{1} & \lambda_{0} & \lambda_{3} & -\lambda_{2} \\
-\lambda_{2} & -\lambda_{3} & \lambda_{0} & \lambda_{1} \\
-\lambda_{3} & \lambda_{2} & -\lambda_{1} & \lambda_{0}
\end{array}\right),
$$

by differentiating with respect to time. The differential equations, determining the rigid body rotation in the terms of the Rodrigues - Hamilton parameters are:

$$
\left(\begin{array}{c}
\ddot{\lambda}_{0} \\
\ddot{\lambda}_{1} \\
\ddot{\lambda}_{2} \\
\ddot{\lambda}_{3}
\end{array}\right)=\frac{1}{2} K^{-1}\left(\begin{array}{c}
\frac{1}{2} \omega^{2} \\
\dot{\omega}_{1} \\
\dot{\omega}_{2} \\
\dot{\omega}_{3}
\end{array}\right), K^{-1}=\left(\begin{array}{cccc}
-\lambda_{0} & -\lambda_{1} & -\lambda_{2} & -\lambda_{3} \\
-\lambda_{1} & \lambda_{0} & -\lambda_{3} & \lambda_{2} \\
-\lambda_{2} & \lambda_{3} & \lambda_{0} & -\lambda_{1} \\
-\lambda_{3} & -\lambda_{2} & \lambda_{1} & \lambda_{0}
\end{array}\right) .
$$

Here $\omega^{2}=|\bar{\omega}|^{2} ; \lambda_{0}^{2}+\lambda_{1}^{2}+\lambda_{2}^{2}+\lambda_{3}^{2}=1 ; \lambda_{0} \dot{\lambda}_{0}+\lambda_{1} \dot{\lambda}_{1}+\lambda_{2} \dot{\lambda}_{2}+\lambda_{3} \dot{\lambda}_{3}=0$. 


\section{CONCLUSIONS}

Thus Lagrange function for the relativistic rotation of the rigid body, which is generated by metric properties of Riemann space of general relativity, was received:

$$
L_{n}=L_{n}^{\text {Newton }}+\Delta L_{n} \text {. }
$$

The differential equations, determining the rigid body rotation in the terms of the Rodrigues Hamilton parameters are derived.

Also are derived the differential equations, which are determining the rigid body rotation in the terms of the Rodrigues - Hamilton parameters:

$$
\left(\begin{array}{c}
\ddot{\lambda}_{0} \\
\ddot{\lambda}_{1} \\
\ddot{\lambda}_{2} \\
\ddot{\lambda}_{3}
\end{array}\right)=\frac{1}{2} K^{-1}\left(\begin{array}{c}
\frac{1}{2} \omega^{2} \\
\dot{\omega}_{1} \\
\dot{\omega}_{2} \\
\dot{\omega}_{3}
\end{array}\right), K^{-1}=\left(\begin{array}{cccc}
-\lambda_{0} & -\lambda_{1} & -\lambda_{2} & -\lambda_{3} \\
-\lambda_{1} & \lambda_{0} & -\lambda_{3} & \lambda_{2} \\
-\lambda_{2} & \lambda_{3} & \lambda_{0} & -\lambda_{1} \\
-\lambda_{3} & -\lambda_{2} & \lambda_{1} & \lambda_{0}
\end{array}\right) .
$$

\section{APPENDIX A}

In the following decompositions of the integrands of Lagrange function (2.3), only the principal terms of the expansion and terms that depend on the angular velocity $\bar{\omega}$ or contain $\bar{\rho}$ not higher and $\bar{\rho}$ not less (2.4) than the second order are retained:

1) $\dot{\bar{R}}_{n}^{* 2}=\dot{\bar{R}}_{n}^{2}+2 \dot{\bar{R}}_{n} \cdot(\bar{\omega} \times \bar{\rho})+(\bar{\omega} \times \bar{\rho})^{2}=\dot{\bar{R}}_{n}^{2}+2 \bar{\rho} \cdot(\dot{\bar{R}} \times \bar{\omega}]+(\bar{\omega} \times \bar{\rho})^{2}$;

2) $\dot{\bar{R}}_{n}^{* 4}=\left[\dot{\bar{R}}_{n}^{2}+2 \bar{\rho} \cdot\left(\dot{\bar{R}}_{n} \times \bar{\omega}\right)+(\bar{\omega} \times \bar{\rho})^{2}\right]\left[\dot{\bar{R}}_{n}^{2}+2 \bar{\rho} \cdot\left(\dot{\bar{R}}_{n} \times \bar{\omega}\right)+(\bar{\omega} \times \bar{\rho})^{2}\right]=$

$$
=\dot{\bar{R}}_{n}^{4}+4 \vec{R}_{n}^{2} \bar{\rho} \cdot\left(\dot{\bar{R}}_{n} \times \bar{\omega}\right)+4\left[\bar{\rho} \cdot\left(\dot{\bar{R}}_{n} \times \bar{\omega}\right)\right]^{2}+2 \dot{\bar{R}}_{n}^{2}(\bar{\omega} \times \bar{\rho})^{2}+\ldots
$$

3) $\frac{1}{\Delta_{n j}^{*}} \frac{1}{\Delta_{n k}^{*}}=\frac{1}{\Delta_{n j}}\left\{1-\frac{\bar{\rho} \cdot\left(\bar{R}_{n}-\bar{R}_{j}\right)}{\Delta_{n j}^{2}}-\frac{\bar{\rho}^{2}}{2 \Delta_{n j}^{2}}+\frac{3}{2} \frac{\left[\bar{\rho} \cdot\left(\bar{R}_{n}-\bar{R}_{j}\right)\right]^{2}}{\Delta_{n j}^{4}}+\ldots\right\}$

$$
\frac{1}{\Delta_{n k}}\left\{1-\frac{\bar{\rho} \cdot\left(\bar{R}_{n}-\bar{R}_{k}\right)}{\Delta_{n k}^{2}}-\frac{\bar{\rho}^{2}}{2 \Delta_{n k}^{2}}+\frac{3}{2} \frac{\left[\bar{\rho} \cdot\left(\bar{R}_{n}-\bar{R}_{k}\right)\right]^{2}}{\Delta_{n k}^{4}}+\ldots\right\}=
$$

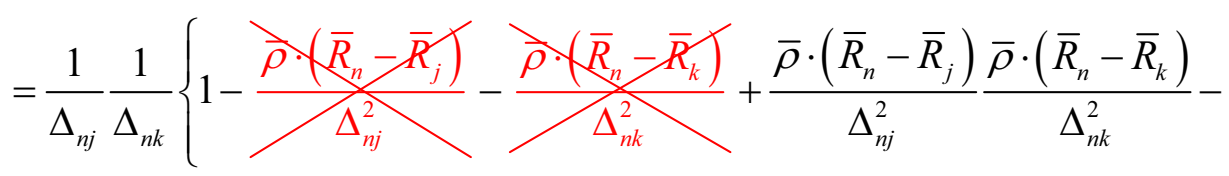$$
\left.-\frac{\bar{\rho}^{2}}{2 \Delta_{n j}^{2}}+\frac{3}{2} \frac{\left[\bar{\rho} \cdot\left(\bar{R}_{n}-\bar{R}_{j}\right)\right]^{2}}{\Delta_{n j}^{4}}-\frac{\bar{\rho}^{2}}{2 \Delta_{n k}^{2}}+\frac{3}{2} \frac{\left[\bar{\rho} \cdot\left(\bar{R}_{n}-\bar{R}_{k}\right)\right]^{2}}{\Delta_{n k}^{4}}+\ldots\right\}
$$

4) $\frac{\dot{\bar{R}}_{n}^{* 2}}{\Delta_{n j}^{*}}=\left[\dot{\bar{R}}_{n}^{2}+2 \bar{\rho} \cdot\left(\dot{\bar{R}}_{n} \times \bar{\omega}\right)+(\bar{\omega} \times \bar{\rho})^{2}\right]\left[\frac{1}{\Delta_{n j}}-\frac{\bar{\rho} \cdot\left(\bar{R}_{n}-\bar{R}_{j}\right)}{\Delta_{n j}^{3}}-\frac{\bar{\rho}^{2}}{2 \Delta_{n j}^{3}}+\frac{3}{2} \frac{\left[\bar{\rho} \cdot\left(\bar{R}_{n}-\bar{R}_{j}\right)\right]^{2}}{\Delta_{n j}^{5}}\right]=$ 


$$
\begin{aligned}
= & \frac{\dot{\bar{R}}_{n}^{2}}{\Delta_{n j}}+2 \frac{\bar{\rho} \cdot\left(\dot{\bar{R}}_{n} \times \widehat{\omega}\right)}{\Delta_{n j}}+\frac{(\bar{\omega} \times \bar{\rho})^{2}}{\Delta_{n j}}-\dot{\bar{R}}_{n}^{2} \frac{\bar{\alpha} \cdot\left(\bar{R}_{n}-\bar{R}_{j}\right)}{\Delta_{n j}^{3}}-2 \frac{\bar{\rho} \cdot\left(\dot{\bar{R}}_{n} \times \bar{\omega}\right) \bar{\rho} \cdot\left(\bar{R}_{n}-\bar{R}_{j}\right)}{\Delta_{n j}^{3}}- \\
& -\dot{\bar{R}}_{n}^{2} \frac{\bar{\rho}^{2}}{2 \Delta_{n j}^{3}}+\dot{\bar{R}}_{n}^{2} \frac{3}{2} \frac{\left[\bar{\rho} \cdot\left(\bar{R}_{n}-\bar{R}_{j}\right)\right]^{2}}{\Delta_{n j}^{5}}+\ldots ;
\end{aligned}
$$

5) $\frac{\dot{\bar{R}}_{n}^{*} \cdot \dot{\bar{R}}_{j}}{\Delta_{n j}^{*}}=\left[\dot{\bar{R}}_{n} \cdot \dot{\bar{R}}_{j}+\bar{\rho} \cdot\left(\dot{\bar{R}}_{j} \times \bar{\omega}\right)\right]\left[\frac{1}{\Delta_{n j}}-\frac{\bar{\rho} \cdot\left(\bar{R}_{n}-\bar{R}_{j}\right)}{\Delta_{n j}^{3}}-\frac{\bar{\rho}^{2}}{2 \Delta_{n j}^{3}}+\frac{3}{2} \frac{\left[\bar{\rho} \cdot\left(\bar{R}_{n}-\bar{R}_{j}\right)\right]^{2}}{\Delta_{n j}^{5}}\right]=$

$$
\begin{aligned}
= & \frac{\dot{\bar{R}}_{n} \cdot \dot{\bar{R}}_{j}}{\Delta_{n j}}+\frac{\bar{\rho} \cdot\left(\dot{\bar{R}}_{j} \times \bar{\omega}\right)}{\Delta_{n j}}+\frac{\bar{R}_{n} \cdot \dot{\bar{R}}_{j} \bar{\rho} \cdot\left(\bar{R}-\bar{R}_{j}\right)}{\Delta_{n j}^{3}}-\frac{\bar{\rho} \cdot\left(\dot{\bar{R}}_{j} \times \bar{\omega}\right) \bar{\rho} \cdot\left(\bar{R}_{n}-\bar{R}_{j}\right)}{\Delta_{n j}^{3}}- \\
& -\frac{\dot{\bar{R}}_{n} \cdot \overline{\bar{R}}_{j} \bar{\rho}^{2}}{2 \Delta_{n j}^{3}}+\frac{3}{2} \frac{\dot{\bar{R}}_{n} \cdot \dot{\bar{R}}_{j}\left[\bar{\rho} \cdot\left(\bar{R}_{n}-\bar{R}_{j}\right)\right]^{2}}{\Delta_{n j}^{5}} ;
\end{aligned}
$$

6) $\frac{1}{\Delta_{n j}^{*}}=\frac{1}{\Delta_{n j}}\left[1+2 \frac{\bar{\rho} \cdot\left(\bar{R}_{n}-\bar{R}_{j}\right)}{\Delta_{n j}^{2}}+\frac{\bar{\rho}^{2}}{\Delta_{n j}^{2}}\right]^{-\frac{1}{2}}=\frac{1}{\Delta_{n j}}-\frac{\bar{\rho} \cdot\left(\bar{R}_{n}-\bar{R}_{j}\right)}{\Delta_{n j}^{3}}-\frac{\bar{\rho}^{2}}{2 \Delta_{n j}^{3}}+\frac{3}{2} \frac{\left[\bar{\rho} \cdot\left(\bar{R}_{n}-\bar{R}_{j}\right)\right]^{2}}{\Delta_{n j}^{5}}+\ldots$;

7) $\dot{\bar{R}}_{n}^{*} \cdot \frac{\left(\bar{R}_{n}^{*}-\bar{R}_{j}\right)}{\Delta_{n j}^{*}} \dot{\bar{R}}_{j} \cdot \frac{\left(\bar{R}_{n}^{*}-\bar{R}_{j}\right)}{\Delta_{n j}^{*}} \frac{1}{\Delta_{n j}^{*}}=\left[\dot{\bar{R}}_{n} \cdot\left(\bar{R}_{n}^{*}-\bar{R}_{j}\right)+(\bar{\omega} \times \bar{\rho}) \cdot\left(\bar{R}_{n}^{*}-\bar{R}_{j}\right)\right] \dot{\bar{R}}_{j} \cdot\left(\bar{R}_{n}^{*}-\bar{R}_{j}\right) \frac{1}{\Delta_{n j}^{* 3}}=$ $=\left\{\dot{\bar{R}}_{n} \cdot\left(\bar{R}_{n}^{*}-\bar{R}_{j}\right)+\bar{\rho} \cdot\left[\left(\bar{R}_{n}^{*}-\bar{R}_{j}\right) \times \bar{\omega}\right]\right\} \dot{\bar{R}}_{j} \cdot\left(\bar{R}_{n}^{*}-\bar{R}_{j}\right) \frac{1}{\Delta_{n j}^{* 3}}=$

$=\left\{\dot{\bar{R}}_{n} \cdot\left(\bar{R}_{n}-\bar{R}_{j}\right)+\bar{\rho} \cdot \dot{\bar{R}}_{n}+\bar{\rho} \cdot\left[\left(\bar{R}_{n}-\bar{R}_{j}\right) \times \bar{\omega}\right]+\bar{\rho} \cdot(\bar{\rho} \times \bar{\omega}) \equiv 0\right\}\left[\dot{\bar{R}}_{j} \cdot\left(\bar{R}_{n}-\bar{R}_{j}\right)+\bar{\rho} \cdot \dot{\bar{R}}_{j}\right]$

$$
\left\{\frac{1}{\Delta_{n j}^{3}}-3 \frac{\bar{\rho} \cdot\left(\bar{R}_{n}-\bar{R}_{j}\right)}{\Delta_{n j}^{5}}-\frac{3}{2} \frac{\bar{\rho}^{2}}{\Delta_{n j}^{5}}+\frac{15}{2} \frac{\left[\bar{\rho} \cdot\left(\bar{R}_{n}-\bar{R}_{j}\right)\right]^{2}}{\Delta_{n j}^{7}}\right\}=
$$

$=\left[\dot{\bar{R}}_{n} \cdot\left(\bar{R}_{n}-\bar{R}_{j}\right) \dot{\bar{R}}_{j} \cdot\left(\bar{R}_{n}-\bar{R}_{j}\right)+\bar{\rho} \cdot \dot{\bar{R}}_{j} \dot{\bar{R}}_{n} \cdot\left(\bar{R}_{n}-\bar{R}_{j}\right)+\bar{\rho} \cdot \dot{\bar{R}}_{n} \dot{\bar{R}}_{j} \cdot\left(\bar{R}_{n}-\bar{R}_{j}\right)+\bar{\rho} \cdot \dot{\bar{R}}_{n} \bar{\rho} \cdot \dot{\bar{R}}_{j}+\right.$

$\left.+\bar{\rho} \cdot\left[\left(\bar{R}_{n}-\bar{R}_{j}\right) \times \bar{\omega}\right] \overline{\bar{R}}_{j} \cdot\left(\bar{R}_{n}-\bar{R}_{j}\right)+\bar{\rho} \cdot\left[\left(\bar{R}_{n}-\bar{R}_{j}\right) \times \bar{\omega}\right] \bar{\rho} \cdot \dot{\bar{R}}_{j}\right]$

$\left\{\frac{1}{\Delta_{n j}^{3}}-3 \frac{\bar{\rho} \cdot\left(\bar{R}_{n}-\bar{R}_{j}\right)}{\Delta_{n j}^{5}}-\frac{3}{2} \frac{\bar{\rho}^{2}}{\Delta_{n j}^{5}}+\frac{15}{2} \frac{\left[\bar{\rho} \cdot\left(\bar{R}_{n}-\bar{R}_{j}\right)\right]^{2}}{\Delta_{n j}^{7}}\right\}=$

$=\dot{\bar{R}}_{n} \cdot\left(\bar{R}_{n}-\bar{R}_{j}\right) \dot{\bar{R}}_{j} \cdot\left(\bar{R}_{n}-\bar{R}_{j}\right) \frac{1}{\Delta_{n j}^{3}}-\overline{\dot{\bar{R}}_{n} \cdot\left(\bar{R}_{n}-\bar{R}_{j}\right) \dot{R}_{j} \cdot\left(\bar{R}_{n}=\overline{R_{j}}\right) 3 \frac{\bar{P}\left(\bar{R}_{n}-R_{j}\right)}{\Delta_{n j}^{5}}-}$

$-\dot{\bar{R}}_{n} \cdot\left(\bar{R}_{n}-\bar{R}_{j}\right) \dot{\bar{R}}_{j} \cdot\left(\bar{R}_{n}-\bar{R}_{j}\right) \frac{3}{2} \frac{\bar{\rho}^{2}}{\Delta_{n j}^{5}}+\dot{\bar{R}}_{n} \cdot\left(\bar{R}_{n}-\bar{R}_{j}\right) \dot{\bar{R}}_{j} \cdot\left(\bar{R}_{n}-\bar{R}_{j}\right) \frac{15}{2} \frac{\left[\bar{\rho} \cdot\left(\bar{R}_{n}-\bar{R}_{j}\right)\right]^{2}}{\Delta_{n j}^{7}}+$

$+\overline{\bar{R}}_{n} \cdot\left(\bar{R}_{n}-\overline{R_{j}}\right) \overline{\bar{\rho} \cdot \dot{R_{j}} \frac{T}{\Delta_{n i}^{3}}}-3 \dot{\bar{R}_{n}} \cdot\left(\bar{R}_{n}-\bar{R}_{j}\right) \bar{\rho} \cdot \dot{\bar{R}_{j}} \frac{\bar{\rho} \cdot\left(\bar{R}_{n}-\bar{R}_{j}\right)}{\Delta_{n j}^{5}}+$ 


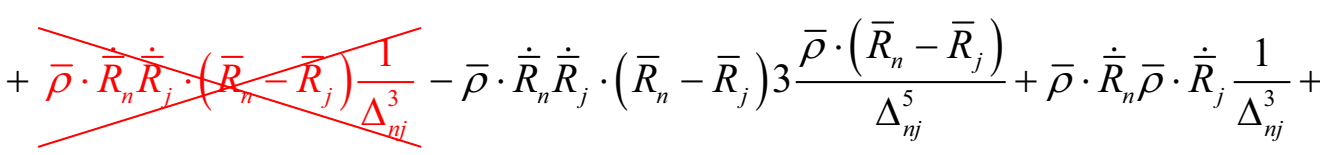

$+\overline{\bar{\rho} \cdot\left[\left(\bar{R}_{n}-\bar{R}_{j}\right) \times \bar{\varphi}\right] \dot{\dot{R}_{j}} \cdot\left(\bar{R}_{n}-\bar{R}_{j}\right) \frac{1}{\Delta_{n j}^{3}}}-\bar{\rho} \cdot\left[\left(\bar{R}_{n}-\bar{R}_{j}\right) \times \bar{\omega}\right] \dot{\bar{R}}_{j} \cdot\left(\bar{R}_{n}-\bar{R}_{j}\right) 3 \frac{\bar{\rho} \cdot\left(\bar{R}_{n}-\bar{R}_{j}\right)}{\Delta_{n j}^{5}}+$

$+\bar{\rho} \cdot\left[\left(\bar{R}_{n}-\bar{R}_{j}\right) \times \bar{\omega}\right] \bar{\rho} \cdot \dot{\bar{R}}_{j} \frac{1}{\Delta_{n j}^{3}}+\ldots$.

To compute some 12 integrals:

$$
\begin{aligned}
& \int_{m_{n}}(\bar{\omega} \times \bar{\rho})^{2} d m_{n}=\int\left[\left(\omega_{m_{n}} \zeta-\omega_{3} \eta\right)^{2}+\left(\omega_{3} \xi-\omega_{1} \zeta\right)^{2}+\left(\omega_{1} \eta-\omega_{2} \xi\right)^{2}\right] d m_{n}= \\
= & {\left[\left(\omega_{1}^{2}+\omega_{2}^{2}\right) \frac{A_{n}+B_{n}-C_{n}}{2}+\left(\omega_{3}^{2}+\omega_{1}^{2}\right) \frac{C_{n}+A_{n}-B_{n}}{2}+\left(\omega_{3}^{2}+\omega_{2}^{2}\right) \frac{\left.B_{n}+C_{n}-A_{n}\right]}{2}\right]=} \\
= & \left(A_{n} \omega_{1}^{2}+B_{n} \omega_{2}^{2}+C_{n} \omega_{3}^{2}\right)=\bar{H}_{n} \cdot \bar{\omega} ; \\
& \int_{m_{n}} \bar{\rho}^{2} d m_{n}=\int_{m_{n}}\left(\xi^{2}+\eta^{2}+\zeta^{2}\right) d m_{n}=\frac{1}{2}\left(A_{n}+B_{n}+C_{n}\right) ; \\
& \int_{m_{n}}\left[\left(\bar{R}_{n}-\bar{R}_{j}\right) \cdot \bar{\rho}\right]^{2} d m_{n}=\int\left[\left(x_{n}-x_{j}\right)^{2} \xi^{2}+\left(y_{n}-y_{j}\right)^{2} \eta^{2}+\left(z_{n}-z_{j}\right)^{2} \zeta^{2}\right] d m_{n}= \\
= & \frac{1}{2}\left[\left(x_{n}-x_{j}\right)^{2}\left(B_{n}+C_{n}-A_{n}\right)+\left(y_{n}-y_{j}\right)^{2}\left(C_{n}+A_{n}-B_{n}\right)+\left(z_{n}-z_{j}\right)^{2}\left(A_{n}+B_{n}-C_{n}\right)\right]= \\
= & \frac{1}{2}\left\{\Delta_{n j}^{2}\left(A_{n}+B_{n}+C_{n}\right)-2\left[\left(x_{n}-x_{j}\right)^{2} A_{n}+\left(y_{n}-y_{j}\right)^{2} B_{n}+\left(z_{n}-z_{j}\right)^{2} C_{n}\right]\right\} ;
\end{aligned}
$$


111

$$
\begin{aligned}
& \int_{m_{n}}\left[\bar{\rho} \cdot\left(\dot{\bar{R}}_{n} \times \bar{\omega}\right)\right]^{2} d m_{n}= \\
& =\int_{m_{n}} \xi^{2}\left(\dot{y}_{n} \omega_{3}-\dot{z}_{n} \omega_{2}\right)^{2} d m_{n}+\int_{m_{n}} \eta^{2}\left(\dot{z}_{n} \omega_{1}-\dot{x}_{n} \omega_{3}\right)^{2} d m_{n}+\int_{m_{n}} \zeta^{2}\left(\dot{x}_{n} \omega_{2}-\dot{y}_{n} \omega_{1}\right)^{2} d m_{n}= \\
& =\left(\dot{y}_{n} \omega_{3}-\dot{z}_{n} \omega_{2}\right)^{2} \frac{B_{n}+C_{n}-A_{n}}{2}+\left(\dot{z}_{n} \omega_{1}-\dot{x}_{n} \omega_{3}\right)^{2} \frac{C_{n}+A_{n}-B_{n}}{2}+\left(\dot{x}_{n} \omega_{2}-\dot{y}_{n} \omega_{1}\right)^{2} \frac{A_{n}+B_{n}-C_{n}}{2} \text {; } \\
& \int_{m_{n}} \bar{\rho} \cdot\left(\dot{\bar{R}}_{n} \times \bar{\omega}\right) \bar{\rho} \cdot\left(\bar{R}_{n}-\bar{R}_{j}\right) d m_{n}=\left[\int_{m_{n}} \xi^{2}\left(\dot{y}_{n} \omega_{3}-\dot{z}_{n} \omega_{2}\right)\left(x_{n}-x_{j}\right) d m_{n}+\right. \\
& \left.+\int_{m_{n}} \eta^{2}\left(\dot{z}_{n} \omega_{1}-\dot{x}_{n} \omega_{3}\right)\left(y_{n}-y_{j}\right) d m_{n}+\int_{m_{n}} \zeta^{2}\left(\dot{x}_{n} \omega_{2}-\dot{y}_{n} \omega_{1}\right)\left(z_{n}-z_{j}\right) d m_{n}\right]= \\
& =\frac{1}{2}\left[\left(\dot{y}_{n} \omega_{3}-\dot{z}_{n} \omega_{2}\right)\left(x_{n}-x_{j}\right)\left(B_{n}+C_{n}-A_{n}\right)+\left(\dot{z}_{n} \omega_{1}-\dot{x}_{n} \omega_{3}\right)\left(y_{n}-y_{j}\right)\left(C_{n}+A_{n}-B_{n}\right)+\right. \\
& \left.+\left(\dot{x}_{n} \omega_{2}-\dot{y}_{n} \omega_{1}\right)\left(z_{n}-z_{j}\right)\left(A_{n}+B_{n}-C_{n}\right)\right]= \\
& =\frac{1}{2}\left\{A_{n} \omega_{1}\left[\left(y_{n}-y_{j}\right) \dot{z}_{n}-\left(z_{n}-z_{j}\right) \dot{y}_{n}\right]+B_{n} \omega_{2}\left[\left(z_{n}-z_{j}\right) \dot{x}_{n}-\left(x_{n}-x_{j}\right) \dot{z}_{n}\right]+\right. \\
& +C_{n} \omega_{3}\left[\left(x_{n}-x_{j}\right) \dot{y}_{n}-\left(y_{n}-y_{j}\right) \dot{x}_{n}\right]+\left(C_{n}-B_{n}\right) \omega_{1}\left[\left(y_{n}-y_{j}\right) \dot{z}_{n}+\left(z_{n}-z_{j}\right) \dot{y}_{n}\right]+ \\
& \left.+\left(A_{n}-C_{n}\right) \omega_{2}\left[\left(z_{n}-z_{j}\right) \dot{x}_{n}+\left(x_{n}-x_{j}\right) \dot{z}_{n}\right]+\left(B_{n}-A_{n}\right) \omega_{3}\left[\left(x_{n}-x_{j}\right) \dot{y}_{n}+\left(y_{n}-y_{j}\right) \dot{x}_{n}\right]\right\}= \\
& =\frac{1}{2}\left\{\bar{H}_{n} \cdot\left(\bar{R}_{n}-\bar{R}_{j}\right) \times \dot{\bar{R}}_{n}+\right. \\
& +\left(C_{n}-B_{n}\right) \omega_{1}\left[\left(y_{n}-y_{j}\right) \dot{z}_{n}+\left(z_{n}-z_{j}\right) \dot{y}_{n}\right]+\left(A_{n}-C_{n}\right) \omega_{2}\left[\left(z_{n}-z_{j}\right) \dot{x}_{n}+\left(x_{n}-x_{j}\right) \dot{z}_{n}\right]+ \\
& \left.+\left(B_{n}-A_{n}\right) \omega_{3}\left[\left(x_{n}-x_{j}\right) \dot{y}_{n}+\left(y_{n}-y_{j}\right) \dot{x}_{n}\right]\right\} \text {; } \\
& \int_{m_{n}} \bar{\rho} \cdot\left(\dot{\bar{R}}_{j} \times \bar{\omega}\right) \bar{\rho} \cdot\left(\bar{R}_{n}-\bar{R}_{j}\right) d m_{n}=\frac{1}{2}\left\{\bar{H}_{n} \cdot\left(\bar{R}_{n}-\bar{R}_{j}\right) \times \dot{\bar{R}}_{j}+\right. \\
& +\left(C_{n}-B_{n}\right) \omega_{1}\left[\left(y_{n}-y_{j}\right) \dot{z}_{j}+\left(z_{n}-z_{j}\right) \dot{y}_{j}\right]+\left(A_{n}-C_{n}\right) \omega_{2}\left[\left(z_{n}-z_{j}\right) \dot{x}_{j}+\left(x_{n}-x_{j}\right) \dot{z}_{j}\right]+ \\
& \left.+\left(B_{n}-A_{n}\right) \omega_{3}\left[\left(x_{n}-x_{j}\right) \dot{y}_{j}+\left(y_{n}-y_{j}\right) \dot{x}_{j}\right]\right\} \text {; } \\
& \int_{m_{n}} \dot{\bar{R}}_{j} \cdot \bar{\rho}\left(\bar{R}_{n}-\bar{R}_{j}\right) \cdot \bar{\rho} d m_{n}=\frac{1}{2}\left[\dot{x}_{j}\left(x_{n}-x_{j}\right)\left(B_{n}+C_{n}-A_{n}\right)+\right. \\
& \left.+\dot{y}_{j}\left(y_{n}-y_{j}\right)\left(C_{n}+A_{n}-B_{n}\right)+\dot{z}_{j}\left(z_{n}-z_{j}\right)\left(A_{n}+B_{n}-C_{n}\right)\right]= \\
& =\frac{\dot{\bar{R}}_{j} \cdot\left(\bar{R}_{n}-\bar{R}_{j}\right)}{2}\left(A_{n}+B_{n}+C_{n}\right)-\left[\dot{x}_{j}\left(x_{n}-x_{j}\right) A_{n}+\dot{y}_{j}\left(y_{n}-y_{j}\right) B_{n}+\dot{z}_{j}\left(z_{n}-z_{j}\right) C_{n}\right] \text {; }
\end{aligned}
$$




$$
\begin{aligned}
& \int_{m_{n}} \dot{\bar{R}}_{n} \cdot \bar{\rho}\left(\bar{R}_{n}-\bar{R}_{j}\right) \cdot \bar{\rho} d m_{n}=\frac{1}{2}\left[\dot{x}_{n}\left(x_{n}-x_{j}\right)\left(B_{n}+C_{n}-A_{n}\right)+\right. \\
& \left.+\dot{y}_{n}\left(y_{n}-y_{j}\right)\left(C_{n}+A_{n}-B_{n}\right)+\dot{z}_{n}\left(z_{n}-z_{j}\right)\left(A_{n}+B_{n}-C_{n}\right)\right]= \\
& =\frac{\dot{\bar{R}}_{n} \cdot\left(\bar{R}_{n}-\bar{R}_{j}\right)}{2}\left(A_{n}+B_{n}+C_{n}\right)-\left[\dot{x}_{n}\left(x_{n}-x_{j}\right) A_{n}+\dot{y}_{n}\left(y_{n}-y_{j}\right) B_{n}+\dot{z}_{n}\left(z_{n}-z_{j}\right) C_{n}\right] \text {; } \\
& \int_{m_{n}} \dot{\bar{R}}_{n} \cdot \bar{\rho} \dot{\bar{R}}_{j} \cdot \bar{\rho} d m_{n}=\frac{1}{2}\left[\dot{x}_{n} \dot{x}_{j}\left(B_{n}+C_{n}-A_{n}\right)+\dot{y}_{n} \dot{y}_{j}\left(C_{n}+A_{n}-B_{n}\right)+\right. \\
& \left.+\dot{z}_{n} \dot{z}_{j}\left(A_{n}+B_{n}-C_{n}\right)\right]=\frac{\dot{\bar{R}}_{n} \cdot \dot{\bar{R}}_{j}}{2}\left(A_{n}+B_{n}+C_{n}\right)-\left[\dot{x}_{n} \dot{x}_{j} A_{n}+\dot{y}_{n} \dot{y}_{j} B_{n}+\dot{z}_{n} \dot{z}_{j} C_{n}\right] \\
& \int_{m_{n}} \bar{\rho} \cdot\left[\left(\bar{R}_{n}-\bar{R}_{j}\right) \times \bar{\omega}\right] \bar{\rho} \cdot\left(\bar{R}_{n}-\bar{R}_{j}\right) d m_{n}=\int_{m_{n}} \xi^{2}\left(x_{n}-x_{j}\right)\left[\left(y_{n}-y_{j}\right) \omega_{3}-\left(z_{n}-z_{j}\right) \omega_{2}\right] d m_{n}+ \\
& +\int_{m_{n}} \eta^{2}\left(y_{n}-y_{j}\right)\left[\left(z_{n}-z_{j}\right) \omega_{1}-\left(x_{n}-x_{j}\right) \omega_{3}\right] d m_{n}+\int_{m_{n}} \zeta^{2}\left(z_{n}-z_{j}\right)\left[\left(x_{n}-x_{j}\right) \omega_{2}-\left(y_{n}-y_{j}\right) \omega_{1}\right] d m_{n}= \\
& =\frac{1}{2}\left\{\left(x_{n}-x_{j}\right)\left[\omega_{3}\left(y_{n}-y_{j}\right)-\omega_{2}\left(z_{n}-z_{j}\right)\right]\left(B_{n}+C_{n}-A_{n}\right)+\right. \\
& +\left(y_{n}-y_{j}\right)\left[\omega_{1}\left(z_{n}-z_{j}\right)-\omega_{3}\left(x_{n}-x_{j}\right)\right]\left(C_{n}+A_{n}-B_{n}\right)+ \\
& \left.+\left(z_{n}-z_{j}\right)\left[\omega_{2}\left(x_{n}-x_{j}\right)-\omega_{1}\left(y_{n}-y_{j}\right)\right]\left(A_{n}+B_{n}-C_{n}\right)\right\}= \\
& =\left(x_{n}-x_{j}\right)\left(y_{n}-y_{j}\right) \omega_{3}\left(B_{n}-A_{n}\right)+\left(z_{n}-z_{j}\right)\left(x_{n}-x_{j}\right) \omega_{2}\left(A_{n}-C_{n}\right)+ \\
& +\left(y_{n}-y_{j}\right)\left(z_{n}-z_{j}\right) \omega_{1}\left(C_{n}-B_{n}\right) \text {; } \\
& \int_{m_{n}} \bar{\rho} \cdot\left(\bar{R}_{n}-\bar{R}_{j}\right) \bar{\rho} \cdot\left(\bar{R}_{n}-\bar{R}_{k}\right) d m_{n}=\frac{1}{2}\left[\left(x_{n}-x_{j}\right)\left(x_{n}-x_{k}\right)\left(B_{n}+C_{n}-A_{n}\right)+\right. \\
& \left.+\left(y_{n}-y_{j}\right)\left(y_{n}-y_{k}\right)\left(C_{n}+A_{n}-B_{n}\right)+\left(z_{n}-z_{j}\right)\left(z_{n}-z_{k}\right)\left(A_{n}+B_{n}-C_{n}\right)\right]= \\
& =\frac{\left(\bar{R}_{n}-\bar{R}_{j}\right) \cdot\left(\bar{R}_{n}-\bar{R}_{k}\right)}{2}\left(A_{n}+B_{n}+C_{n}\right)- \\
& -\left[A_{n}\left(x_{n}-x_{j}\right)\left(x_{n}-x_{k}\right)+B_{n}\left(y_{n}-y_{j}\right)\left(y_{n}-y_{k}\right)+C_{n}\left(z_{n}-z_{j}\right)\left(z_{n}-z_{k}\right)\right] \text {; }
\end{aligned}
$$




$$
\begin{aligned}
& \int_{m_{n}} \bar{\rho} \cdot\left[\left(\bar{R}_{n}-\bar{R}_{j}\right) \times \bar{\omega}\right] \bar{\rho} \cdot \dot{\bar{R}}_{j} d m_{n}=\int_{m_{n}} \xi^{2} \dot{x}_{j}\left[\left(y_{n}-y_{j}\right) \omega_{3}-\left(z_{n}-z_{j}\right) \omega_{2}\right] d m_{n}+ \\
+ & \int_{m_{n}} \eta^{2} \dot{y}_{j}\left[\left(z_{n}-z_{j}\right) \omega_{1}-\left(x_{n}-x_{j}\right) \omega_{3}\right] d m_{n}+\int_{m_{n}} \zeta^{2} \dot{z}_{j}\left[\left(x_{n}-x_{j}\right) \omega_{2}-\left(y_{n}-y_{j}\right) \omega_{1}\right] d m_{n}= \\
= & \frac{1}{2}\left\{\dot{x}_{j}\left[\omega_{3}\left(y_{n}-y_{j}\right)-\omega_{2}\left(z_{n}-z_{j}\right)\right]\left(B_{n}+C_{n}-A_{n}\right)+\right. \\
+ & \left.\dot{y}_{j}\left[\omega_{1}\left(z_{n}-z_{j}\right)-\omega_{3}\left(x_{n}-x_{j}\right)\right]\left(C_{n}+A_{n}-B_{n}\right)+\dot{z}_{j}\left[\omega_{2}\left(x_{n}-x_{j}\right)-\omega_{1}\left(y_{n}-y_{j}\right)\right]\left(A_{n}+B_{n}-C_{n}\right)\right\}= \\
= & \frac{1}{2}\left\{A_{n} \omega_{1}\left[\dot{y}_{j}\left(z_{n}-z_{j}\right)-\dot{z}_{j}\left(y_{n}-y_{j}\right)\right]+B_{n} \omega_{2}\left[\dot{z}_{j}\left(x_{n}-x_{j}\right)-\dot{x}_{j}\left(z_{n}-z_{j}\right)\right]+\right. \\
+ & C_{n} \omega_{3}\left[\dot{x}_{j}\left(y_{n}-y_{j}\right)-\dot{y}_{j}\left(x_{n}-x_{j}\right)\right]+\left(C_{n}-B_{n}\right) \omega_{1}\left[\dot{y}_{j}\left(z_{n}-z_{j}\right)+\dot{z}_{j}\left(y_{n}-y_{j}\right)\right]+ \\
+ & \left.\left(A_{n}-C_{n}\right) \omega_{2}\left[\dot{z}_{j}\left(x_{n}-x_{j}\right)+\dot{x}_{j}\left(z_{n}-z_{j}\right)\right]+\left(B_{n}-A_{n}\right) \omega_{3}\left[\dot{x}_{j}\left(y_{n}-y_{j}\right)+\dot{y}_{j}\left(x_{n}-x_{j}\right)\right]\right\}= \\
= & \frac{1}{2}\left\{\bar{H}_{n} \cdot \dot{\bar{R}}_{j} \times\left(\bar{R}_{n}-\bar{R}_{j}\right)+\left(C_{n}-B_{n}\right) \omega_{1}\left[\dot{y}_{j}\left(z_{n}-z_{j}\right)+\dot{z}_{j}\left(y_{n}-y_{j}\right)\right]+\right. \\
+ & \left.\left(A_{n}-C_{n}\right) \omega_{2}\left[\dot{z}_{j}\left(x_{n}-x_{j}\right)+\dot{x}_{j}\left(z_{n}-z_{j}\right)\right]+\left(B_{n}-A_{n}\right) \omega_{3}\left[\dot{x}_{j}\left(y_{n}-y_{j}\right)+\dot{y}_{j}\left(x_{n}-x_{j}\right)\right]\right\} .
\end{aligned}
$$

\section{APPENDIX B}

The partial derivatives of the kinetic energy are computed by using (1.1):

$$
\begin{aligned}
& \frac{\partial T}{\partial \lambda_{i}}=A_{n} \omega_{1} \frac{\partial \omega_{1}}{\partial \lambda_{i}}+B_{n} \omega_{2} \frac{\partial \omega_{2}}{\partial \lambda_{i}}+C_{n} \omega_{3} \frac{\partial \omega_{3}}{\partial \lambda_{i}}, \\
& \frac{\partial \omega_{1}}{\partial \lambda_{0}}=2 \dot{\lambda}_{1} ; \frac{\partial \omega_{1}}{\partial \lambda_{1}}=-2 \dot{\lambda}_{0} ; \frac{\partial \omega_{1}}{\partial \lambda_{2}}=-2 \dot{\lambda}_{3} ; \frac{\partial \omega_{1}}{\partial \lambda_{3}}=2 \dot{\lambda}_{2} ; \\
& \frac{\partial \omega_{2}}{\partial \lambda_{0}}=2 \dot{\lambda}_{2} ; \frac{\partial \omega_{2}}{\partial \lambda_{1}}=2 \dot{\lambda}_{3} ; \frac{\partial \omega_{2}}{\partial \lambda_{2}}=-2 \dot{\lambda}_{0} ; \frac{\partial \omega_{2}}{\partial \lambda_{3}}=-2 \dot{\lambda}_{1} ; \\
& \frac{\partial \omega_{3}}{\partial \lambda_{0}}=2 \dot{\lambda}_{3} ; \frac{\partial \omega_{3}}{\partial \lambda_{1}}=-2 \dot{\lambda}_{2} ; \frac{\partial \omega_{3}}{\partial \lambda_{2}}=2 \dot{\lambda}_{1} ; \frac{\partial \omega_{3}}{\partial \lambda_{3}}=-2 \dot{\lambda}_{0} ; \\
& \frac{\partial T}{\partial \dot{\lambda}_{i}}=A_{n} \omega_{1} \frac{\partial \omega_{1}}{\partial \dot{\lambda}_{i}}+B_{n} \omega_{2} \frac{\partial \omega_{2}}{\partial \dot{\lambda}_{i}}+C_{n} \omega_{3} \frac{\partial \omega_{3}}{\partial \dot{\lambda}_{i}}, \\
& \frac{\partial \omega_{1}}{\partial \dot{\lambda}_{0}}=-2 \lambda_{1} ; \frac{\partial \omega_{1}}{\partial \dot{\lambda}_{1}}=2 \lambda_{0} ; \frac{\partial \omega_{1}}{\partial \dot{\lambda}_{2}}=2 \lambda_{3} ; \frac{\partial \omega_{1}}{\partial \dot{\lambda}_{3}}=-2 \lambda_{2} ; \\
& \frac{\partial \omega_{2}}{\partial \dot{\lambda}_{0}}=-2 \lambda_{2} ; \frac{\partial \omega_{2}}{\partial \dot{\lambda}_{1}}=-2 \lambda_{3} ; \frac{\partial \omega_{2}}{\partial \dot{\lambda}_{2}}=2 \lambda_{0} ; \frac{\partial \omega_{2}}{\partial \dot{\lambda}_{3}}=2 \lambda_{1} ; \\
& \frac{\partial \omega_{3}}{\partial \dot{\lambda}_{0}}=-2 \lambda_{3} ; \frac{\partial \omega_{3}}{\partial \dot{\lambda}_{1}}=2 \lambda_{2} ; \frac{\partial \omega_{3}}{\partial \dot{\lambda}_{2}}=-2 \lambda_{1} ; \frac{\partial \omega_{3}}{\partial \dot{\lambda}_{3}}=2 \lambda_{0} ;
\end{aligned}
$$




$$
\begin{aligned}
& \frac{d}{d t} \frac{\partial T}{\partial \dot{\lambda}_{i}}=A_{n} \dot{\omega}_{1} \frac{\partial \omega_{1}}{\partial \dot{\lambda}_{i}}+B_{n} \dot{\omega}_{2} \frac{\partial \omega_{2}}{\partial \dot{\lambda}_{i}}+C_{n} \dot{\omega}_{3} \frac{\partial \omega_{3}}{\partial \dot{\lambda}_{i}}+A_{n} \omega_{1} \frac{d}{d t} \frac{\partial \omega_{1}}{\partial \dot{\lambda}_{i}}+B_{n} \omega_{2} \frac{d}{d t} \frac{\partial \omega_{2}}{\partial \dot{\lambda}_{i}}+C_{n} \omega_{3} \frac{d}{d t} \frac{\partial \omega_{3}}{\partial \dot{\lambda}_{i}} \\
& \frac{d}{d t} \frac{\partial \omega_{1}}{\partial \dot{\lambda}_{0}}=-2 \dot{\lambda}_{1} ; \frac{d}{d t} \frac{\partial \omega_{1}}{\partial \dot{\lambda}_{1}}=2 \dot{\lambda}_{0} ; \frac{d}{d t} \frac{\partial \omega_{1}}{\partial \dot{\lambda}_{2}}=2 \dot{\lambda}_{3} ; \frac{d}{d t} \frac{\partial \omega_{1}}{\partial \dot{\lambda}_{3}}=-2 \dot{\lambda}_{2} ; \\
& \frac{d}{d t} \frac{\partial \omega_{2}}{\partial \dot{\lambda}_{0}}=-2 \dot{\lambda}_{2} ; \frac{d}{d t} \frac{\partial \omega_{2}}{\partial \dot{\lambda}_{1}}=-2 \dot{\lambda}_{3} ; \frac{d}{d t} \frac{\partial \omega_{2}}{\partial \dot{\lambda}_{2}}=2 \dot{\lambda}_{0} ; \frac{d}{d t} \frac{\partial \omega_{2}}{\partial \dot{\lambda}_{3}}=2 \dot{\lambda}_{1} ; \\
& \frac{d}{d t} \frac{\partial \omega_{3}}{\partial \dot{\lambda}_{0}}=-2 \dot{\lambda}_{3} ; \frac{d}{d t} \frac{\partial \omega_{3}}{\partial \dot{\lambda}_{1}}=2 \dot{\lambda}_{2} ; \frac{d}{d t} \frac{\partial \omega_{3}}{\partial \dot{\lambda}_{2}}=-2 \dot{\lambda}_{1} ; \frac{d}{d t} \frac{\partial \omega_{3}}{\partial \dot{\lambda}_{3}}=2 \dot{\lambda}_{0} ; \\
& \text { Thus } \frac{d}{d t} \frac{\partial \omega_{j}}{\partial \dot{\lambda}_{i}}=-\frac{\partial \omega_{j}}{\partial \lambda_{i}}, i=1,2,3,4 ; j=1,2,3 . \\
& \{T\}_{0}=2\left(-\lambda_{1} A_{n} \dot{\omega}_{1}-\lambda_{2} B_{n} \dot{\omega}_{2}-\lambda_{3} C_{n} \dot{\omega}_{3}\right)+4\left(-\dot{\lambda}_{1} A_{n} \omega_{1}-\dot{\lambda}_{2} B_{n} \omega_{2}-\dot{\lambda}_{3} C_{n} \omega_{3}\right) ; \\
& \{T\}_{1}=2\left(\lambda_{0} A_{n} \dot{\omega}_{1}-\lambda_{3} B_{n} \dot{\omega}_{2}+\lambda_{2} C_{n} \dot{\omega}_{3}\right)+4\left(\dot{\lambda}_{0} A_{n} \omega_{1}-\dot{\lambda}_{3} B_{n} \omega_{2}+\dot{\lambda}_{2} C_{n} \omega_{3}\right) ; \\
& \{T\}_{2}=2\left(\lambda_{3} A_{n} \dot{\omega}_{1}+\lambda_{0} B_{n} \dot{\omega}_{2}-\lambda_{1} C_{n} \dot{\omega}_{3}\right)+4\left(\dot{\lambda}_{3} A_{n} \omega_{1}+\dot{\lambda}_{0} B_{n} \omega_{2}-\dot{\lambda}_{1} C_{n} \omega_{3}\right) ; \\
& \{T\}_{3}=2\left(-\lambda_{2} A_{n} \dot{\omega}_{1}+\lambda_{1} B_{n} \dot{\omega}_{2}+\lambda_{0} C_{n} \dot{\omega}_{3}\right)+4\left(-\dot{\lambda}_{2} A_{n} \omega_{1}+\dot{\lambda}_{1} B_{n} \omega_{2}+\dot{\lambda}_{0} C_{n} \omega_{3}\right) .
\end{aligned}
$$

The partial derivatives of the angular momentum vector of the rotational motion of the rigid body are computed:

$$
\begin{aligned}
& \frac{\partial \bar{H}}{\partial \lambda_{i}}=A_{n} \frac{\partial \omega_{1}}{\partial \lambda_{i}} \bar{i}_{1}+B_{n} \frac{\partial \omega_{2}}{\partial \lambda_{i}} \bar{i}_{2}+C_{n} \frac{\partial \omega_{3}}{\partial \lambda_{i}} \bar{i}_{3}, \\
& \frac{\partial \bar{H}}{\partial \dot{\lambda}_{i}}=A_{n} \frac{\partial \omega_{1}}{\partial \dot{\lambda}_{i}} \bar{i}_{1}+B_{n} \frac{\partial \omega_{2}}{\partial \dot{\lambda}_{i}} \bar{i}_{2}+C_{n} \frac{\partial \omega_{3}}{\partial \dot{\lambda}_{i}} \bar{i}_{3}, \\
& \frac{d}{d t} \frac{\partial \bar{H}}{\partial \dot{\lambda}_{i}}=A_{n} \frac{d}{d t} \frac{\partial \omega_{1}}{\partial \dot{\lambda}_{i}} \bar{i}_{1}+B_{n} \frac{d}{d t} \frac{\partial \omega_{2}}{\partial \dot{\lambda}_{i}} \bar{i}_{2}+C_{n} \frac{d}{d t} \frac{\partial \omega_{3}}{\partial \dot{\lambda}_{i}} \bar{i}_{3}, \\
& \frac{d}{d t} \frac{\partial \bar{H}}{\partial \dot{\lambda}_{i}}=-\frac{\partial \bar{H}}{\partial \lambda_{i}} ; \\
& \{\bar{H}\}_{i}=-2\left(A_{n} \frac{\partial \omega_{1}}{\partial \lambda_{i}} \bar{i}_{1}+B_{n} \frac{\partial \omega_{2}}{\partial \lambda_{i}} \bar{i}_{2}+C_{n} \frac{\partial \omega_{3}}{\partial \lambda_{i}} \bar{i}_{3}\right) .
\end{aligned}
$$

Acknowledgements. The investigation was carried out at the Central (Pulkovo) Astronomical Observatory of the Russian Academy of Science (CAO) and the Space Research Centre of the Polish Academy of Science (SRC), under a financial support of the cooperation between SRC and CAO, and Aleksander Brzeziński and Jolanta Nastula personal grants. The authors are grateful to Jose M Ferrandiz for very useful remarks and suggestions.

\section{REFERENCES}

Suslov G.K. (1946): Theoretical mechanics. OGIZ, Moscow, (in Russian).

MacMillan W.D. (1936): Dynamics of rigid bodies, New Yotk and London.

Landau L.D. and Lifshitz E.M. (1967): The Classical Theory of Fields, Moscow: "Nauka". (in Russian) 
Eroshkin G.I., Pashkevich V.V. (1997): Numerical simulation of the rotation motion of the Earth and Moon, Dynamics and Astrometry of Natural and Artificial Celestial Bodies, Proceedings of IAU Colloquium 165 (I.M. Wytrzyszczak, J.H. Lieske, R.A. Feldman eds.), Kluwer, Dordrecht, pp. 275-281.

Pashkevich V.V. (2000): Construction of the High-Precision Numerical Theory of the Rigid Earth Rotation, Artificial Satellites, Journal of Planetary Geodesy, Vol. 35, No 2, pp. $59-71$.

Received: 2018-01-02,

Reviewed: 2018-02-07, 2018-05-07, and 2018-08-27, by Jose M. Ferrandiz Leal, Accepted: 2018-09-03. 